\title{
Direct Activation of NADPH Oxidase 2 by 2-Deoxyribose-1-Phosphate Triggers Nuclear Factor Kappa B-Dependent Angiogenesis
}

\author{
Dina Vara,, Joanna M. Watt, Tiago M. Fortunato, Harry Mellor,, Matthew Burgess, ${ }^{4}$ Kate Wicks, \\ Kimberly Mace, Shaun Reeksting, ${ }^{6}$ Anneke Lubben, ${ }^{6}$ Caroline P.D. Wheeler-Jones, and Giordano Pula ${ }^{1}$
}

\begin{abstract}
Aims: Deoxyribose-1-phosphate (dRP) is a proangiogenic paracrine stimulus released by cancer cells, platelets, and macrophages and acting on endothelial cells. The objective of this study was to clarify how dRP stimulates angiogenic responses in human endothelial cells.

Results: Live cell imaging, electron paramagnetic resonance, pull-down of dRP-interacting proteins, followed by immunoblotting, gene silencing of different NADPH oxidases (NOXs), and their regulatory cosubunits by small interfering RNA (siRNA) transfection, and experiments with inhibitors of the sugar transporter glucose transporter 1 (GLUT1) were utilized to demonstrate that dRP acts intracellularly by directly activating the endothelial NOX2 complex, but not NOX4. Increased reactive oxygen species generation in response to NOX2 activity leads to redox-dependent activation of the transcription factor nuclear factor kappa B (NF- $\kappa \mathrm{B})$, which, in turn, induces vascular endothelial growth factor receptor 2 (VEGFR2) upregulation. Using endothelial tube formation assays, gene silencing by siRNA, and antibody-based receptor inhibition, we demonstrate that the activation of NF- $\kappa \mathrm{B}$ and VEGFR2 is necessary for the angiogenic responses elicited by dRP. The upregulation of VEGFR2 and NOX2-dependent stimulation of angiogenesis by dRP were confirmed in excisional wound and Matrigel plug vascularization assays in vivo using $\mathrm{NOX}^{-/-}$mice.
\end{abstract}

Innovation: For the first time, we demonstrate that dRP acts intracellularly and stimulates superoxide anion generation by direct binding and activation of the NOX2 enzymatic complex.

Conclusions: This study describes a novel molecular mechanism underlying the proangiogenic activity of dRP, which involves the sequential activation of NOX2 and NF- $\kappa \mathrm{B}$ and upregulation of VEGFR2. Antioxid. Redox Signal. 28, 110-130.

Keywords: angiogenesis, endothelial, NADPH, NF- $\kappa$ B, NOX, ROS

\footnotetext{
${ }^{1}$ Institute of Biomedical and Clinical Science, University of Exeter Medical School, Exeter, United Kingdom.

${ }^{2}$ Department of Pharmacy and Pharmacology, University of Bath, Bath, United Kingdom.

${ }^{3}$ Department of Biomedical Engineering, Eindhoven University of Technology, Eindhoven, The Netherlands.

${ }_{5}^{4}$ Department of Biochemistry, University of Bristol, Bristol, United Kingdom.

${ }^{5}$ The Healing Foundation Centre, University of Manchester, Manchester, United Kingdom.

${ }^{6}$ Mass Spectrometry Service and Chemical Characterisation and Analysis Facility, University of Bath, Bath, United Kingdom.

${ }^{7}$ Department of Comparative Biomedical Sciences, Royal Veterinary College, London, United Kingdom.

This work was presented in a reduced form at the ESM-EVBO Conference from May 29, 2017 to June 2, 2017 in Geneva, Switzerland, and the NADPH Oxidase Gordon Conference from June 5, 2016 to June 10, 2016 in Waterville, Maine.

(C) Dina Vara et al., 2017; Published by Mary Ann Liebert, Inc. This is an Open Access article distributed under the terms of the Creative Commons Attribution License, which permits unrestricted use, distribution, and reproduction in any medium, provided the original work is properly cited.
} 


\section{Innovation}

A novel paracrine signaling pathway is described in this study, whereby the angiogenic stimulus 2-deoxyribose-1phosphate (dRP) released by platelets, macrophages, and cancer cells is internalized by endothelial cells and acts intracellularly by directly activating NADPH oxidase 2 (NOX2). In turn, this leads to nuclear factor kappa B (NF$\kappa \mathrm{B})$-dependent upregulation of vascular endothelial growth factor receptor 2 (VEGFR2) and VEGF-dependent angiogenesis. Our elucidation of the mechanism of action of dRP is critical for understanding the full potential of this proangiogenic paracrine signal, which may find important applications in regenerative, vascular, and cancer medicine.

\section{Introduction}

A NGIOGENESIS IS CRITICAL for tissue revascularization and repair after injury. Reactive oxygen species (ROS) have been shown to drive tissue repair by triggering angiogenesis (32). The most important of such factors is vascular endothelial growth factor (VEGF), which is upregulated as a consequence of hypoxia-induced factor-1 (HIF-1) activation $(1,28)$. The increase in ROS triggered by hypoxia and other tissue injuries is associated with oxidation of biological molecules such as lipids and proteins, which has profound effects on cellular physiology (23). Among the sources of ROS associated with the stimulation of angiogenesis, NADPH oxidases (or NOXs) have been studied extensively $(65,69)$. The precise links between NOX activation and angiogenesis remain unclear, but several molecular mechanisms have been implicated, including activation of nitric oxide synthase (13) and VEGF upregulation (32, 69). Endothelial cells express NOX1, NOX2, NOX4, and NOX5 (18). Among NOXs, NOX4 has been shown to lead to stabilization of HIF-1, which in turn stimulates increased transcription of VEGF and drives angiogenesis (69). Other factors, including insulin and TGF- $\beta 1$, also stimulate angiogenesis in an NOX4-dependent manner $(41,46)$. Similarly to NOX4, NOX2 is abundantly expressed in endothelial cells and mediates angiogenesis in response to lipopolysaccharide (LPS) and VEGF $(19,40)$. In keeping with their roles as positive regulators of angiogenesis, both NOX2 and NOX4 can drive endothelial cell migration and capillary-like tube formation in hyperoxic conditions (45). In addition to their effects on the HIF/VEGF signaling axis, NOXs have been shown to stimulate angiogenesis through diverse signaling pathways. For example, activation of nuclear factor kappa $B$ $(\mathrm{NF}-\kappa \mathrm{B})$ downstream of NOX2 (39) or nuclear factor erythroid 2-related factor 2 (Nrf-2) downstream of NOX4 (57) has been shown to be involved in stimulating the angiogenic responses of endothelial cells.

Deoxyribose-1-phosphate (dRP) has previously been described as an endogenous molecule capable of stimulating angiogenesis in an ROS-dependent manner both in vitro and in vivo $(7,24,42,49,50,58)$. The generation of dRP in eukaryotic cells is catalyzed by phosphorylases with specificity for different nucleosides. Three main enzymes have been characterized: thymidine phosphorylase (TP), uridine phosphorylase (UP), and purine nucleoside phosphorylase (PNP) (48). Nucleoside phosphorylases play a key role in nucleoside and pentose metabolism by degrading nucleosides into free nitrogen base and dRP, with dRP converted to deoxyribose-5-phosphate by phosphopentomutase (64). Several studies have suggested that nucleoside phosphorylases stimulate cancer angiogenesis in solid tumors and participate in the progression of the disease $(27,31,62)$. Although regulation of nucleoside phosphorylases is largely unknown and their constitutive activity has been described (5), we previously presented data on the release of dRP by human platelets in response to cellular stimulation (67). In this study, we have evaluated the proangiogenic activity of dRP on human umbilical vein endothelial cells (HUVECs) in vitro using a variety of molecular techniques and have identified the NOX2-NF- $\kappa \mathrm{B}$ signaling axis that is engaged by $\mathrm{dRP}$, resulting in the upregulation of VEGF receptor 2 (VEGFR2) expression and stimulation of angiogenic responses. This study is the most comprehensive and exhaustive characterization of $\mathrm{dRP}$ as a proangiogenic stimulus to date.

FIG. 1. dRP stimulates angiogenesis and oxidative stress of endothelial cells in vitro. (A) The concentration of dRP released by human platelets and mouse macrophages in vitro was quantified by LC-MS. Presented data are from six and three independent samples, respectively. Statistical significance was assessed by one-way ANOVA with Bonferroni post hoc test $\left(* p<0.01\right.$ compared with nonstimulated platelets). (B) HUVECs were seeded at a density of $3 \times 10^{2}$ cells $/ \mathrm{mm}^{2}$ on growth factor-reduced Matrigel ${ }^{\circledR}$ and cultured in basal medium (no FBS). Different concentrations of dRP between $2 \mu M$ and $1 \mathrm{~m} M$ and after $4 \mathrm{~h}$ of culture and quantification of tube number per optical field were performed using the Angiogenesis Analyzer plug-in of ImageJ. (C) ROS generation was analyzed with DHE staining for $1 \mathrm{~h}$ in response to concentrations of dRP ranging from $2 \mu M$ to $1 \mathrm{~m} M$ and expressed as fold increase over basal level. (D) Time course of ROS generation in response to $200 \mu M \mathrm{dRP}$ in the presence of ROS scavengers ( $1 \mathrm{~m} M$ NAC, $10 \mu M$ MnTBAP, or $10 \mu M$ Tempol) or vehicle. ROS production was assessed after 5, 30, 60, and $120 \mathrm{~min}$ and expressed as fold increase over basal level. (E) HUVECs were seeded at a density of $3 \times 10^{2}$ cells $/ \mathrm{mm}^{2}$ on growth factor-reduced Matrigel and cultured in basal medium (no FBS). Two hundred micromolars of dRP was incubated in the presence or absence of $1 \mathrm{~m} M$ NAC, $10 \mu M$ Tempol, or $10 \mu M$ MnTBAP. After $4 \mathrm{~h}$ of culture, quantification of tube number per optical field was performed using the Angiogenesis Analyzer plug-in of ImageJ. Representative pictures are shown (i) and quantification is shown (ii). Throughout the figure, data are expressed as mean \pm SEM and analyzed by one-way ANOVA (B, C) $(n=6)$; $(\mathbf{E})(n=8)$; or two-way ANOVA (D) $(n=6)$. In either case, Bonferroni post hoc test was used to identify statistically significant differences between conditions; ${ }^{*} p<0.05$ compared with vehicle, $* * p<0.05$ compared with dRP. (E) Bars $=300 \mu \mathrm{m}$. ANOVA, analysis of variance; DHE, dihydroethidium; dRP, deoxyribose-1-phosphate; FBS, fetal bovine serum; HUVECs, human umbilical vein endothelial cells; LC-MS, liquid chromatography-mass spectrometry; MnTBAP, Mn(III)tetrakis(4-benzoic acid)porphyrin; NAC, N-acetyl-Lcysteine; ROS, reactive oxygen species; SEM, standard error of the mean; Tempol, 4-hydroxy-2,2,6,6-tetramethylpiperidin-1oxyl. To see this illustration in color, the reader is referred to the web version of this article at www.liebertpub.com/ars 
Understanding the molecular mechanisms underlying the actions of $\mathrm{dRP}$ as a proangiogenic stimulus will have important applications in cancer, vascular, and regenerative medicine.

\section{Results}

dRP stimulates increased levels of ROS generation in an NOX-dependent manner

We have previously described the release of dRP by human platelets (67). Using a quantitative liquid chromatographymass spectrometry (LC-MS) method, we quantified dRP re- leased by human platelets and mouse macrophages. In platelet suspensions at physiological density (i.e., $3 \times 10^{8} / \mathrm{ml}$ ) and in culture medium from confluent murine macrophages, $\mathrm{dRP}$ reached concentrations above $10 \mu M$ (Fig. 1A). The ability of $\mathrm{dRP}$ to induce the formation of capillary-like structures by endothelial cells in vitro (i.e., endothelial tubes) was confirmed for concentrations as low as $2 \mu M$ using low serum and growth factor-reduced Matrigel ${ }^{\circledR}$ (Fig. 1B and Supplementary Fig. S1A; Supplementary Data are available online at www.liebertpub.com/ars), while other pentoses were not effective (Supplementary Fig. S2). We also confirmed that
A

\begin{tabular}{l|l|l} 
Cell type & Stimulus & dRP release $(\mu \mathrm{M})$ \\
\hline \multirow{3}{*}{ Platelets } & None (vehicle) & $8.34 \pm 1.83$ \\
& Thrombin & $9.00 \pm 1.07$ \\
& Collagen & $14.27 \pm 1.00^{*}$ \\
\hline \multirow{3}{*}{ Macrophages } & None (vehicle) & $6.71 \pm 1.59$ \\
& LPS + IFNy & $3.12 \pm 0.30$ \\
& IL4 + IFNy & $13.76 \pm 3.38^{*}$ \\
\hline
\end{tabular}

C

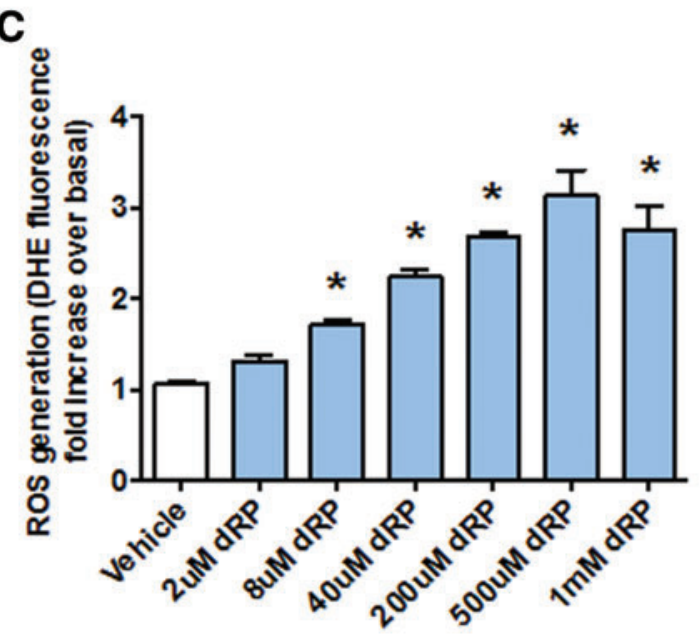

E(i)

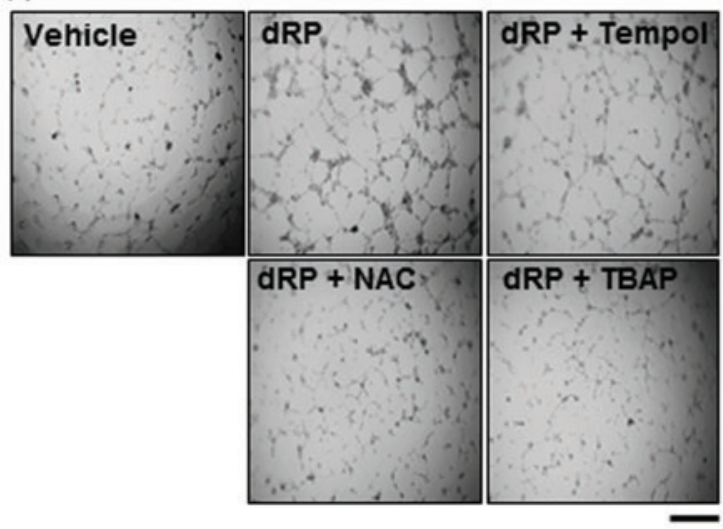

B
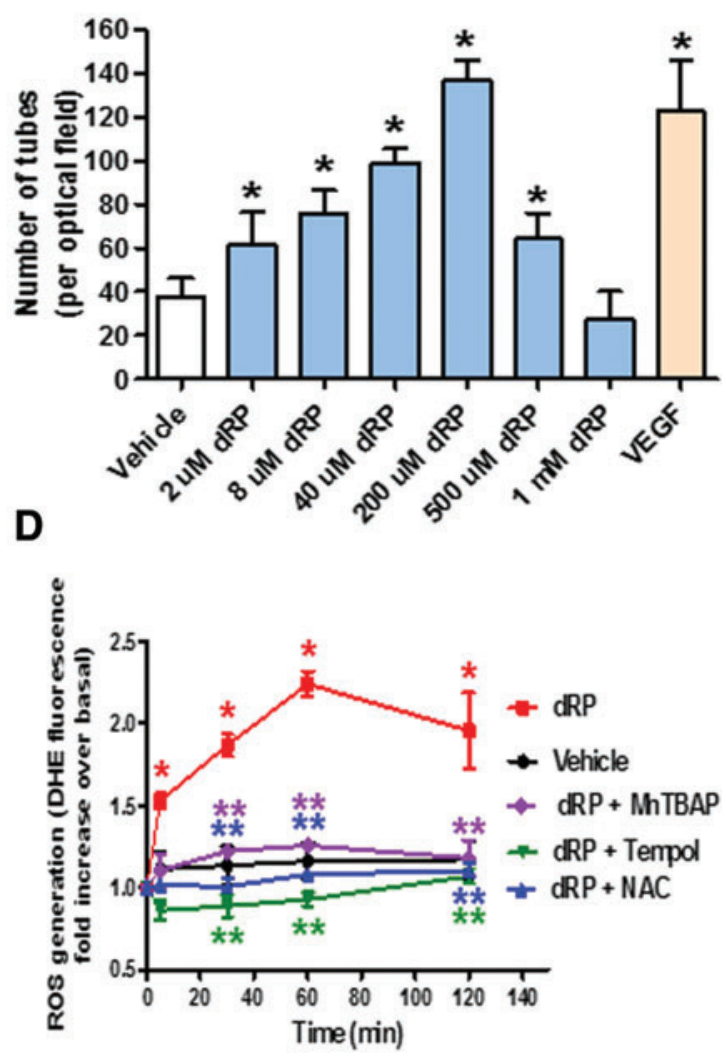

(ii)

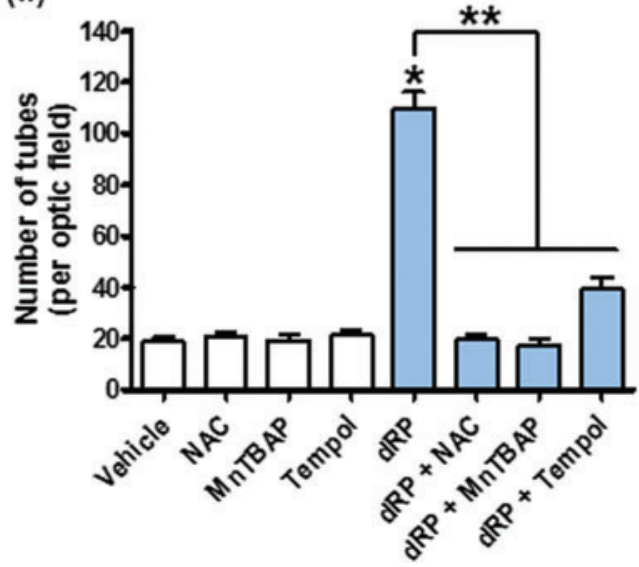


dRP concentrations as low as $8 \mu M$ stimulate a significant increase in endothelial cell ROS formation (while $2 \mu M \mathrm{dRP}$ produced a trend toward increased ROS formation without reaching statistical significance), as measured using dihydroethidium (DHE) after $1 \mathrm{~h}$ of treatment (Fig. 1C). Complete time courses of ROS generation at low micromolar dRP concentrations are shown in Supplementary Figure S1B. The dRP-dependent increase in ROS generation rates was abolished in the presence of $1 \mathrm{~m} M \mathrm{~N}$-acetyl-L-cysteine (NAC), $10 \mu M \mathrm{Mn}$ (III)tetrakis(4-benzoic acid)porphyrin (MnTBAP), or $10 \mu M$ 4-hydroxy-2,2,6,6-tetramethylpiperidin-1-oxyl (Tempol) (Fig. 1D). The link between oxidative stress and angiogenic activity of HUVECs and the role of ROS generation in the angiogenic response induced by $\mathrm{dRP}$ were then tested using the ROS scavenger NAC (71) and the superoxide dismutase (SOD) mimetics, MnTBAP (21) and Tempol (33). All three significantly impaired the tubulogenic activity of dRP (Fig. 1E). Other angiogenic responses induced by dRP (i.e., endothelial cell proliferation and monolayer scratch healing) were also inhibited by NAC, MnTBAP, and Tempol (Supplementary Fig. S3). Interestingly, the stimulatory effect of VEGF on endothelial tube formation (Supplementary Fig. S4) and monolayer scratch healing (Supplementary Fig. S5) was abolished by NOX inhibitors (e.g., pan-NOX inhibitor VAS2870 and the NOX2-specific inhibitor peptide Nox2dstat), but not ROS scavengers (e.g., NAC) and SOD mimetics (e.g., MnTBAP).

Next, we tested the effect of dRP on redox homeostasis in HUVECs by electron paramagnetic resonance (EPR) using the superoxide-specific spin probe 3-methoxycarbonyl-2,2,5,5tetramethylpyrrolidine ( $\mathrm{CMH}$ ) (Fig. 2). In these experiments, we detected a significant increase in the superoxide anion generation rate in response to $\mathrm{dRP}$ from $9.7 \pm 2.9$ to $41.3 \pm 4.6 \mathrm{pmol} \mathrm{min} \mathrm{mg}^{-1} \mathrm{mg}^{-1}$ of cell protein (mean \pm standard error of the mean; shown in Fig. 2A with calibration curve shown in Fig. 2C). Superoxide anion generation detected by EPR was inhibited by the SOD mimetic MnTBAP and by the pan-NOX inhibitor VAS2870 (Fig. 2B). The involvement of NOXs was also confirmed by coimmunoprecipitation of p47phox with NOX2 in the presence of dRP, but not in its absence (Fig. 2D).

\section{dRP directly stimulates NOX2}

EPR analysis remains laborious and time consuming, which limits its application for large numbers of samples. Therefore, we also performed ROS generation analysis using live cell imaging with DHE, which confirmed dRP-induced ROS production in HUVECs. We then explored the source(s) of ROS generated in response to dRP. Treatment with the pan-NOX inhibitor VAS2870 $(1 \mu M)$ suppressed the dRPinduced increase in ROS generation (Fig. 3A). The specific inhibitory peptide Nox2ds-tat (54) was used to show that NOX2 is responsible for the stimulation of ROS generation by dRP. Treatment with Nox2ds-tat completely abolished the dRP-dependent increase in ROS generation in endothelial cells (Fig. 3B). Genetic silencing of NOX2 (i.e., gp91phox subunit) also totally inhibited the dRP-dependent increase in the ROS generation rate (Fig. 3C) and endothelial tube formation (Fig. 3D). In contrast, genetic silencing of NOX4 did not significantly impair dRP-dependent ROS generation (Fig. 3E) or endothelial tube formation (Fig. 3F).
To test whether dRP acts intracellularly, we treated HUVECs for $30 \mathrm{~min}$ with $200 \mu M \mathrm{dRP}$. Following cell disruption by ultracentrifugation, the cytoplasmic fractions were analyzed using LC-MS for the presence of dRP. Interestingly, dRP appeared in the cytoplasm of HUVECs after treatment (Fig 4A). As dRP is administered as a salt, the counter ion cyclohexylammonium was utilized as a control and was found only in the culture media, never in cytoplasmic fractions (data not shown). This suggested the existence of a specific transporter for dRP. Previous studies indicated that the transporter, GLUT1, is the endothelial transporter for several monosaccharides besides hexoses, including riboses (60). To test the hypothesis that GLUT1 is responsible for the internalization of dRP, we utilized two specific inhibitors of this transporter: fasentin and STF-31 $(10 \mu M)$. These inhibitors abolished the ability of dRP to stimulate endothelial tube formation on Matrigel, without affecting VEGF-stimulated tube formation (Fig. 4B). GLUT1 was then silenced by small interfering RNA (siRNA), which resulted in significant inhibition of dRP-dependent ROS generation (Fig. 4C) and tube formation (Fig. 4D). These data suggest that GLUT1 plays a significant role in the internalization and proangiogenic activity of dRP. VEGF-dependent tube formation was not affected by depletion of GLUT1 (Fig. 4D).

Direct binding of dRP to the NOX2 complex (but not to NOX4 or NOX1) was then demonstrated by pull-down experiments using biotinylated dRP and streptavidin beads. In these experiments, dRP was conjugated with biotin in a reaction leading to a mixture of three different adducts (C3-Olinked, C5-O-linked, and C1-OP-linked; Fig 5A). NOX2 (but not NOX4 or NOX1) was selectively pulled down in the presence of biotinylated $\mathrm{dRP}$, but not in the presence of nonbiotinylated dRP. The ability of dRP to activate NOXs directly without the mediation of intracellular signaling pathways was then investigated using a cell-free superoxide anion generation assay (9). Following cell fractionation, HUVEC cytosolic and membrane fractions were characterized for the expression of NOX1, NOX2, and NOX4. All three NOXs are expressed in the membrane fraction, but not in the cytoplasmic fraction (Fig. 5B). Caveolin-1 and actin were used as membrane and cytoplasmic markers, respectively. Upon treatment with dRP, HUVEC membrane fractions induced a significant increase in ferrous cytochrome c compared with membrane fractions treated with vehicle alone. The formation of ferrous cytochrome $\mathrm{c}$ was inhibited in the presence of SOD and by the nonspecific flavoenzyme inhibitor diphenylene iodonium (DPI). The $\mathrm{dRP}$-dependent response registered in this assay was also inhibited by the highly specific peptide inhibitor Nox 2 ds-tat (54), which has been used for membrane-based cell-free assays previously (14). Taken together, these data suggest that dRP is able to directly activate NOX2 without mediation of other signaling events.

To confirm the oxidative status induced by dRP, biotinyliodoacetamide (BIAM) and horseradish peroxidase (HRP)streptavidin were utilized to stain free thiols in HUVEC proteins. These experiments demonstrated that a 4-h exposure to $200 \mu M \mathrm{dRP}$ induces cysteine oxidation (i.e., formation of sulfenic acid and/or disulfide bonds), which appears as a loss of BIAM staining (Fig. 6A). Several bands disappear or become significantly fainter upon treatment with dRP (green arrows in Fig. 6A), which suggests that this molecule induces 
A (i)

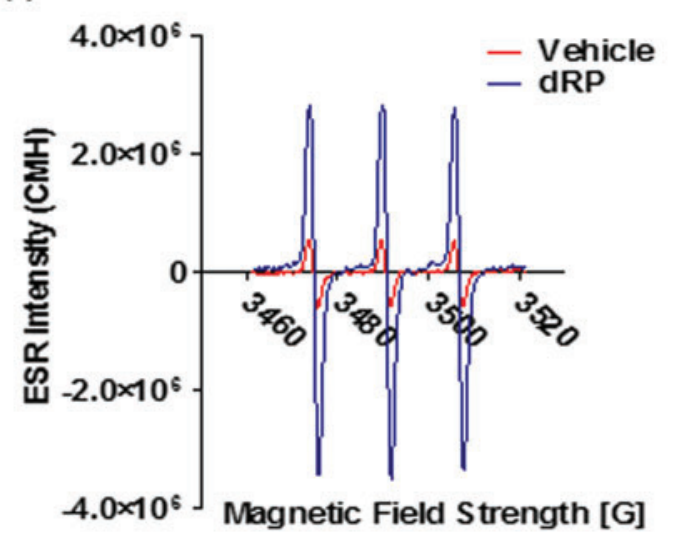

B (i)

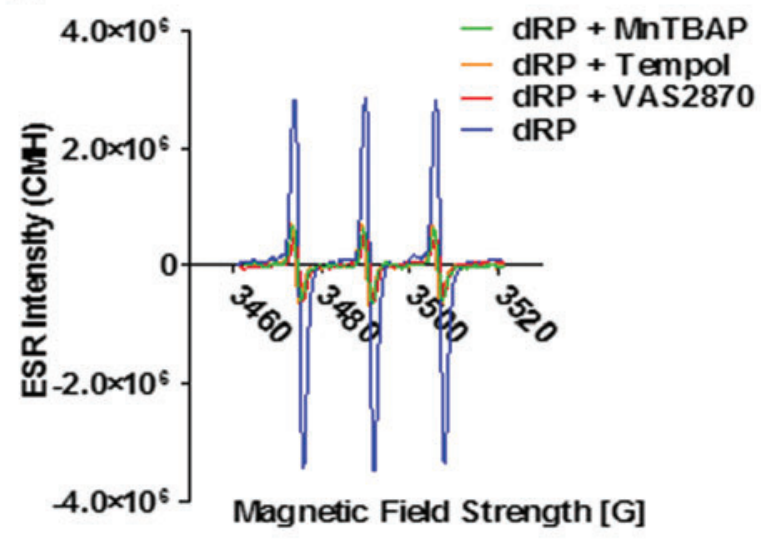

C

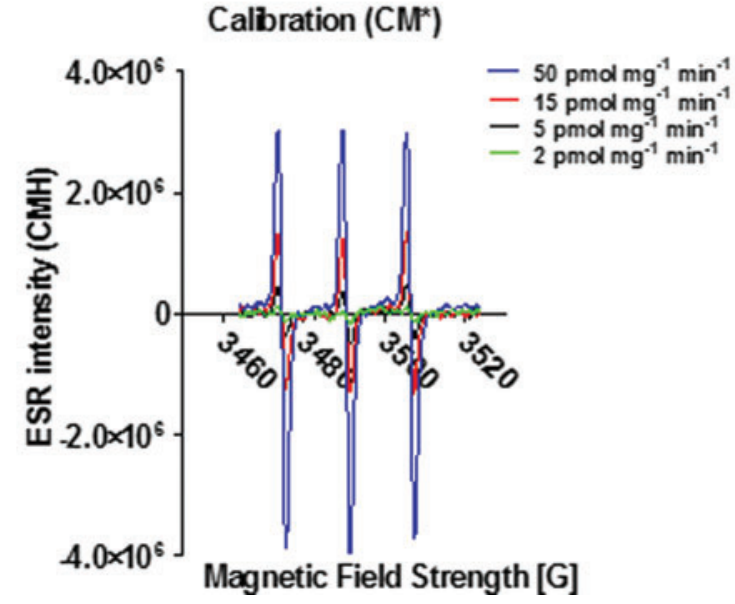

(ii)

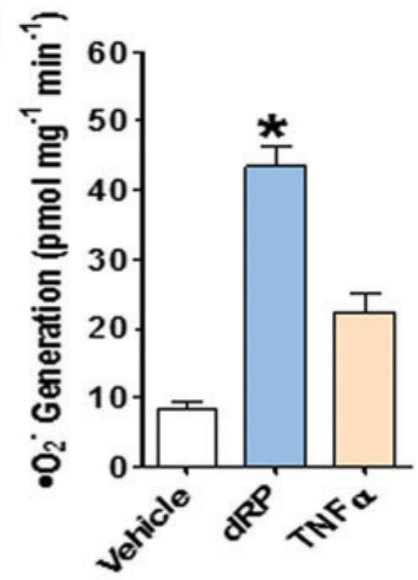

(ii)

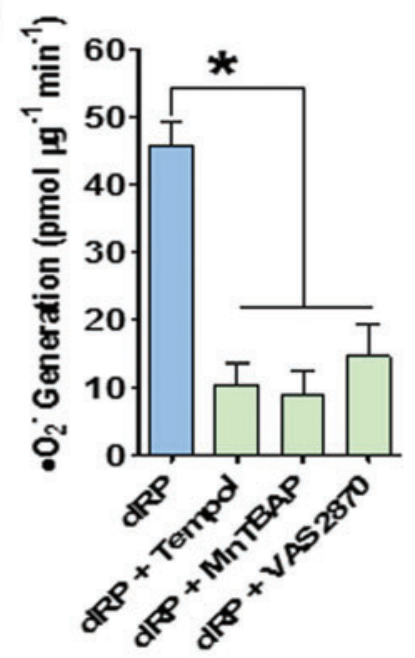

D

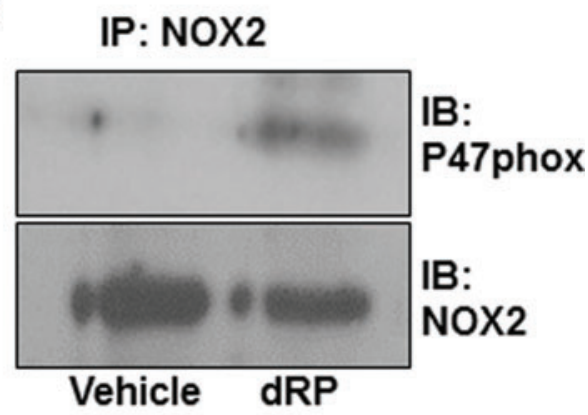

FIG. 2. dRP stimulates increased levels of ROS generation in HUVECs in an NOX-dependent manner. Quantitative measurements of superoxide anion production in HUVECs were performed using the cell-permeable superoxide-specific spin probe CMH and EPR. (A) Cells were treated for 45 min with $200 \mu M$ dRP, vehicle (Tyrode's HEPES buffer), or 50 ng/ $\mathrm{ml} \mathrm{TNF}-\alpha$ in the presence of CMH $(200 \mu M)$ before EPR analysis. (B) Inhibition of superoxide anion production induced by $200 \mu M$ dRP was also detected by EPR by $10 \mu M$ MnTBAP, $10 \mu M$ Tempol, or $1 \mu M$ VAS2870. (A, B) Representative EPR traces are shown (i). The bar charts (ii) show superoxide anion production rates $\left(\mathrm{pmol} \mathrm{mg}^{-1} \mathrm{~min}^{-1}\right.$ ) (mean $\pm \mathrm{SEM}$, one-way ANOVA with Bonferroni post hoc test, $\left.{ }^{*} p<0.05, n=4\right)$. (C) Calibration curve obtained using known concentrations of the oxidized spin probe (i.e., CM*). (D) Activation of NOX2 confirmed by coimmunoprecipitation with p47phox. HUVECs were treated with a vehicle or $200 \mu M \mathrm{dRP}$ for $1 \mathrm{~h}$. NOX2 immunoprecipitates were subjected to immunoblotting for p47phox and NOX2. Blots are representative of four independent experiments. CMH, 3-methoxycarbonyl-2,2,5,5tetramethylpyrrolidine; EPR, electron paramagnetic resonance; NOX, NADPH oxidase. To see this illustration in color, the reader is referred to the web version of this article at www.liebertpub.com/ars 
A

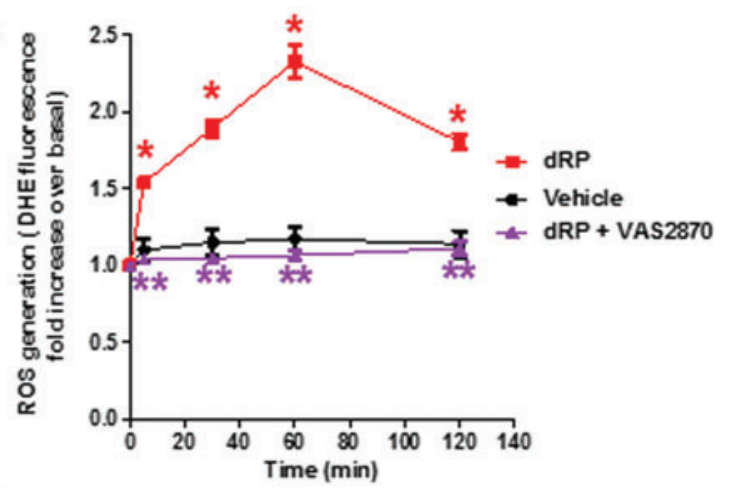

C
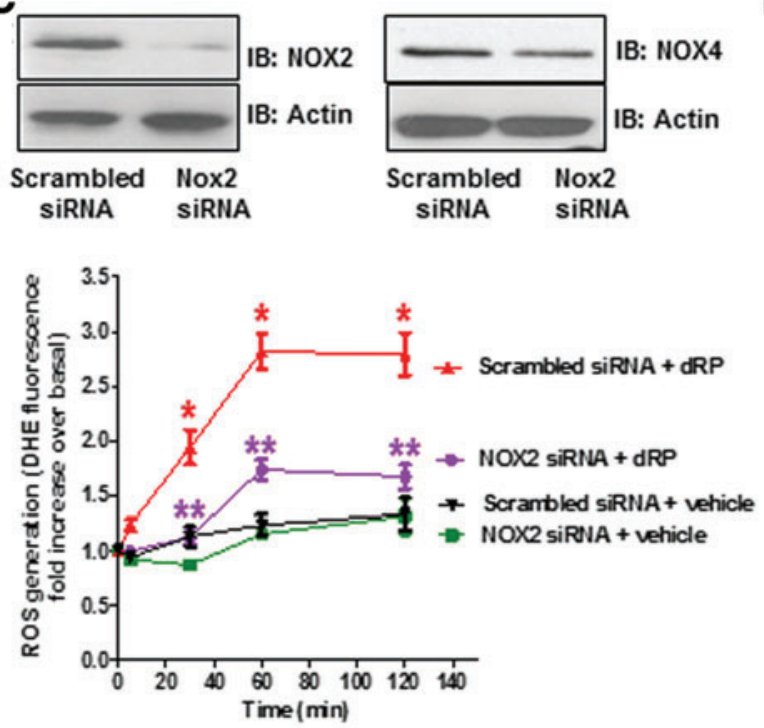

$\mathbf{E}$
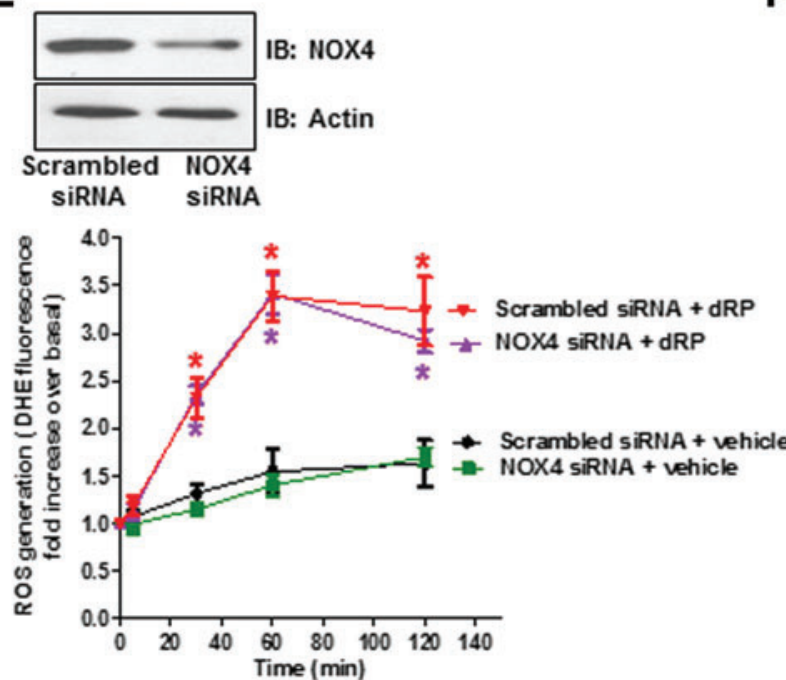

B

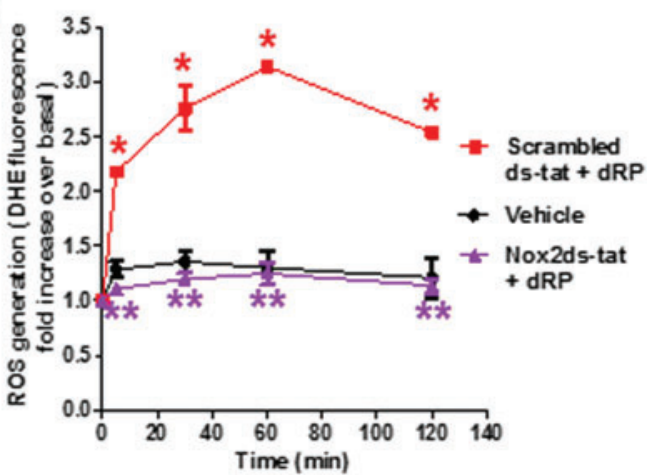

D
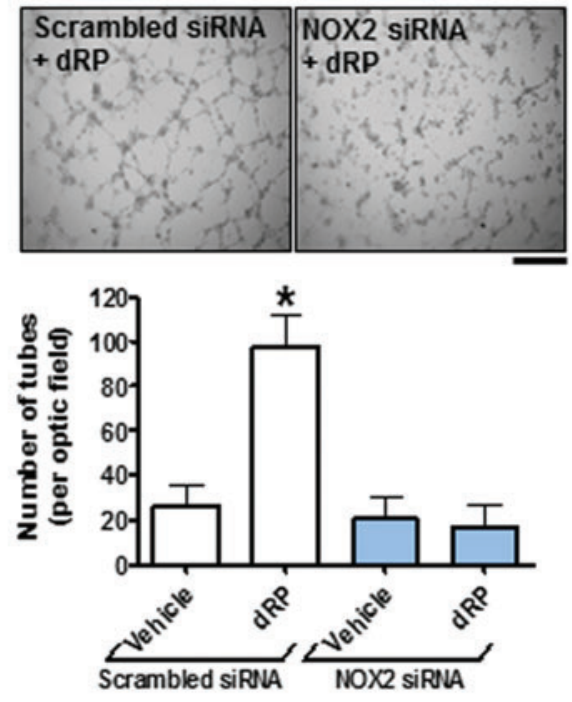

$\mathbf{F}$
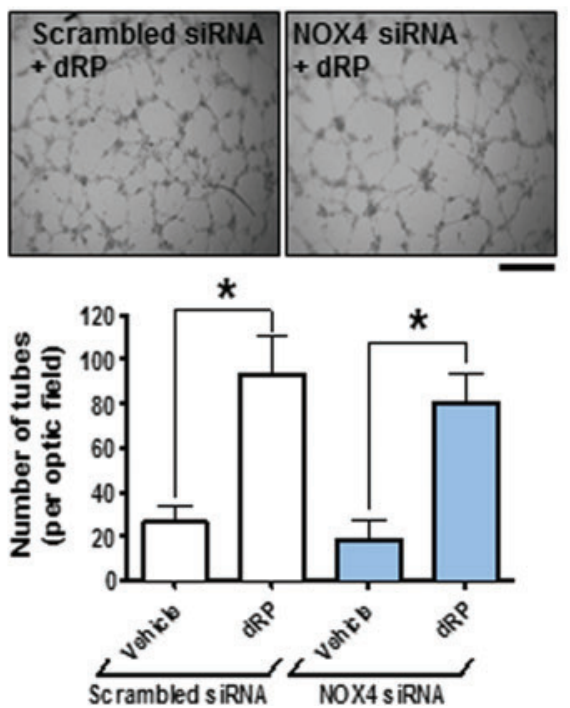

FIG. 3. dRP stimulates increased levels of ROS generation and tube formation in an NOX-dependent manner. (A) ROS generation in response to $200 \mu M$ dRP or vehicle was measured as described above in the presence of $1 \mu M$ VAS2870. (B) dRPinduced ROS production suppressed by inhibition of NOX2 with $10 \mu M$ Nox2ds-tat. Immunoblot analysis of NOX2-specific knockdown by siRNA (and scrambled siRNA control). (C) ROS generation in response to $200 \mu M \mathrm{dRP}$ was measured in control (scrambled siRNA) and NOX2 knockdown cells. (D) Representative images of tube formation by cells transfected with scrambled siRNA and NOX2 siRNA in the presence of $200 \mu M$ dRP are shown. Total number of tubes measured with the Angiogenesis Analyzer plug-in of ImageJ. Representative images (top) and data analysis are shown (bottom). (E, F) Genetic silencing of NOX4 in HUVECs does not affect dRP-induced ROS generation increase. Tube formation in response to $200 \mu M \mathrm{dRP}$ by scrambled and NOX4 knockdown cells was measured. Time courses $(\mathbf{A}-\mathbf{C}, \mathbf{E})$ were analyzed by two-way ANOVA $(n=4)$ with Bonferroni post hoc test $\left[{ }^{*} p<0.05\right.$, compared with vehicle $(\mathbf{A}, \mathbf{B})$ or vehicle/scrambled siRNA $(\mathbf{C}, \mathbf{E})$; $* * p<0.05$ compared with dRP $(\mathbf{A}, \mathbf{B})$ or dRP/ scrambled siRNA (C, E)]. Bar graphs (D, F) represent quantification of tube number per optical field compared by one-way ANOVA with Bonferroni post-test ( ${ }^{*} p<0.05$ compared with vehicle, $\left.n=5\right)$. Bar: (D, F) $300 \mu \mathrm{m}$. siRNA, small interfering RNA. To see this illustration in color, the reader is referred to the web version of this article at www.liebertpub.com/ars 
A

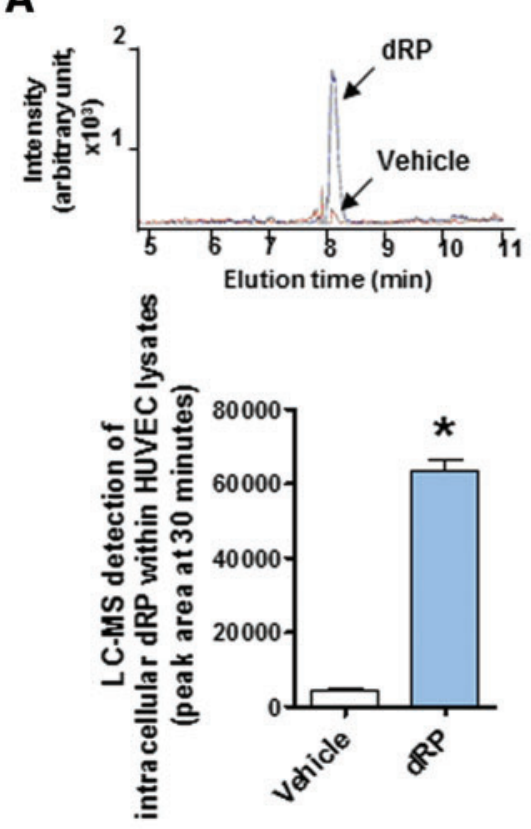

C

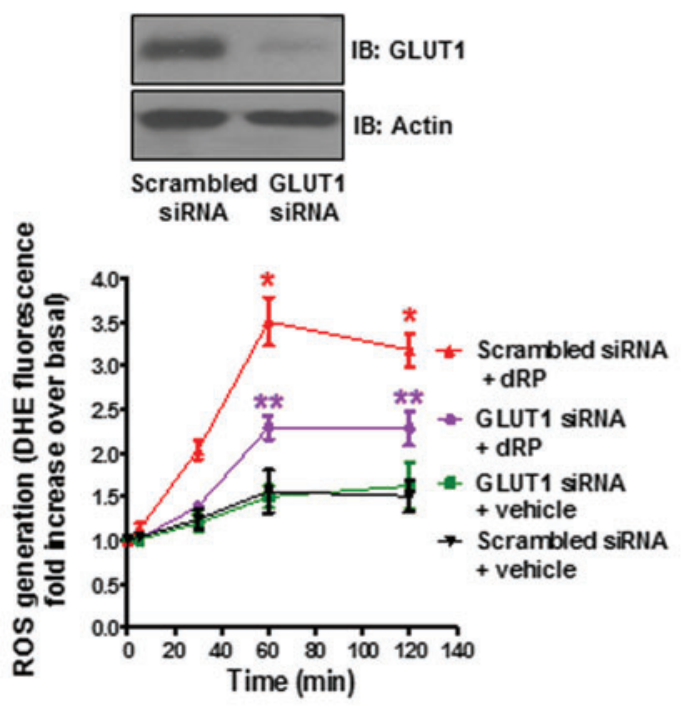

B
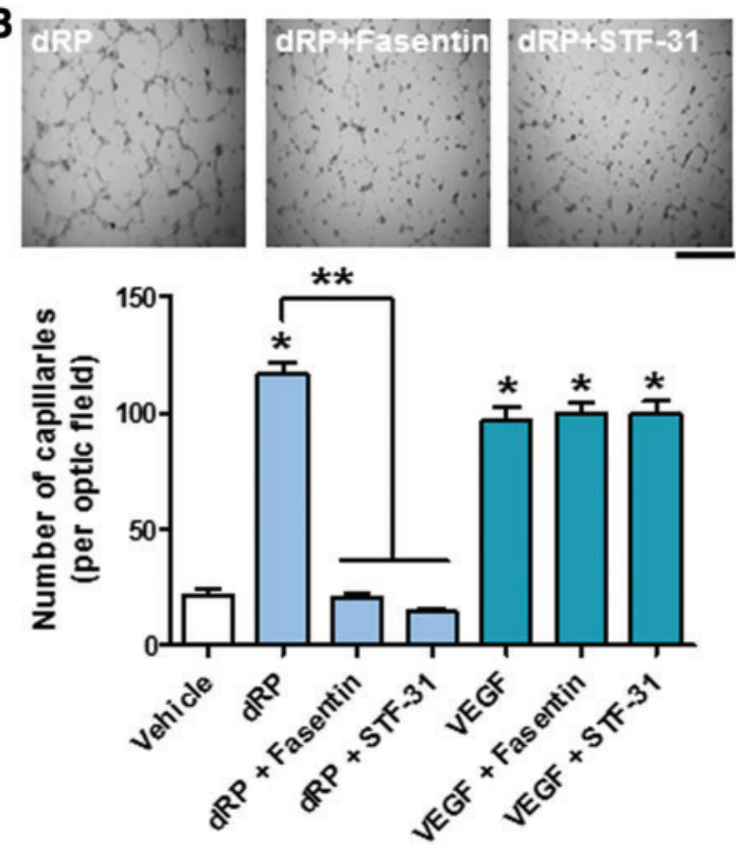

D
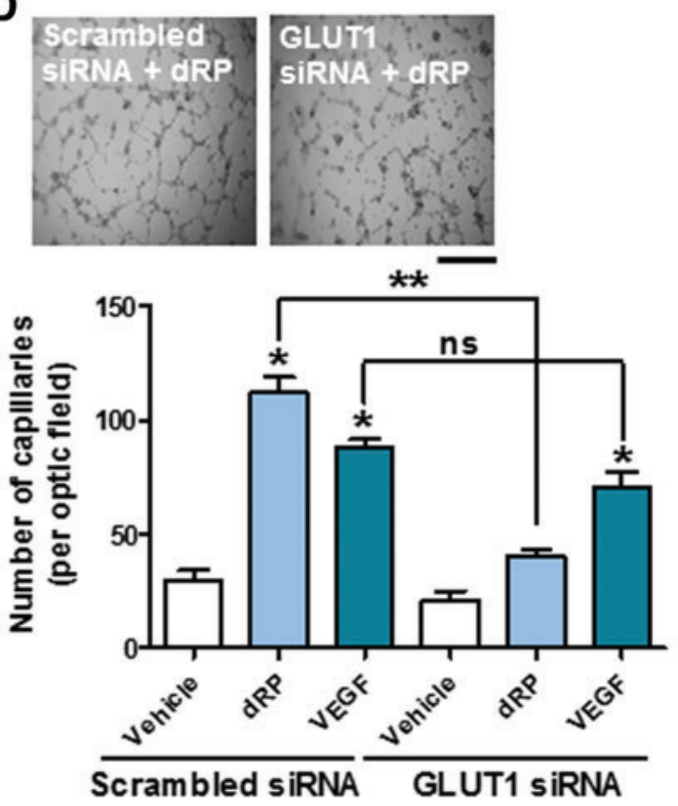

FIG. 4. dRP acts intracellularly following its internalization by the transporter GLUT1. (A) LC-MS detection of intracellular dRP. HUVECs were treated with $200 \mu M$ dRP, before three washes in PBS and ultrasonication. Example chromatogram (upper panel) and data quantification (lower panel) are shown. Statistical significance was tested by $t$-test $\left({ }^{*} p<0.05\right.$, compared with vehicle, $\left.n=4\right)$. (B) STF-31 and fasentin $(10 \mu M)$ inhibited dRP-dependent, but not VEGFdependent, tube formation. Example pictures (upper panel) and data quantification (lower panel) are shown. Bar graphs represent quantification of tube number per optical field compared by one-way ANOVA with Bonferroni post hoc test $(* p<0.05$ compared with vehicle, $n=5)$. Bar: $300 \mu \mathrm{m}$. (C) siRNA-dependent silencing of GLUT1 inhibits dRP-induced ROS generation. Following GLUT1 silencing displayed in top panels, ROS was measured as described over a period of $2 \mathrm{~h}$. Time courses were analyzed by two-way ANOVA $(n=4)$ with Bonferroni post hoc test $(* p<0.05$, compared with vehicle/ scrambled siRNA; **p< 0.05 compared with dRP/scrambled siRNA). (D) siRNA-dependent silencing of GLUT1 inhibits dRP-induced tube formation. Example pictures for dRP response by HUVECs treated with scrambled siRNA or GLUT1 siRNA are shown in top panels. Bar graphs represent quantification of tube number per optical field compared by one-way ANOVA with Bonferroni post hoc test $(* p<0.05$ compared with scrambled siRNA/vehicle, $* * p<0.05$ compared with scrambled siRNA/dRP, ns, nonsignificant, $n=4)$. Bar: $300 \mu \mathrm{m}$. GLUT1, glucose transporter 1; PBS, phosphate-buffered saline; VEGF, vascular endothelial growth factor. To see this illustration in color, the reader is referred to the web version of this article at www.liebertpub.com/ars 

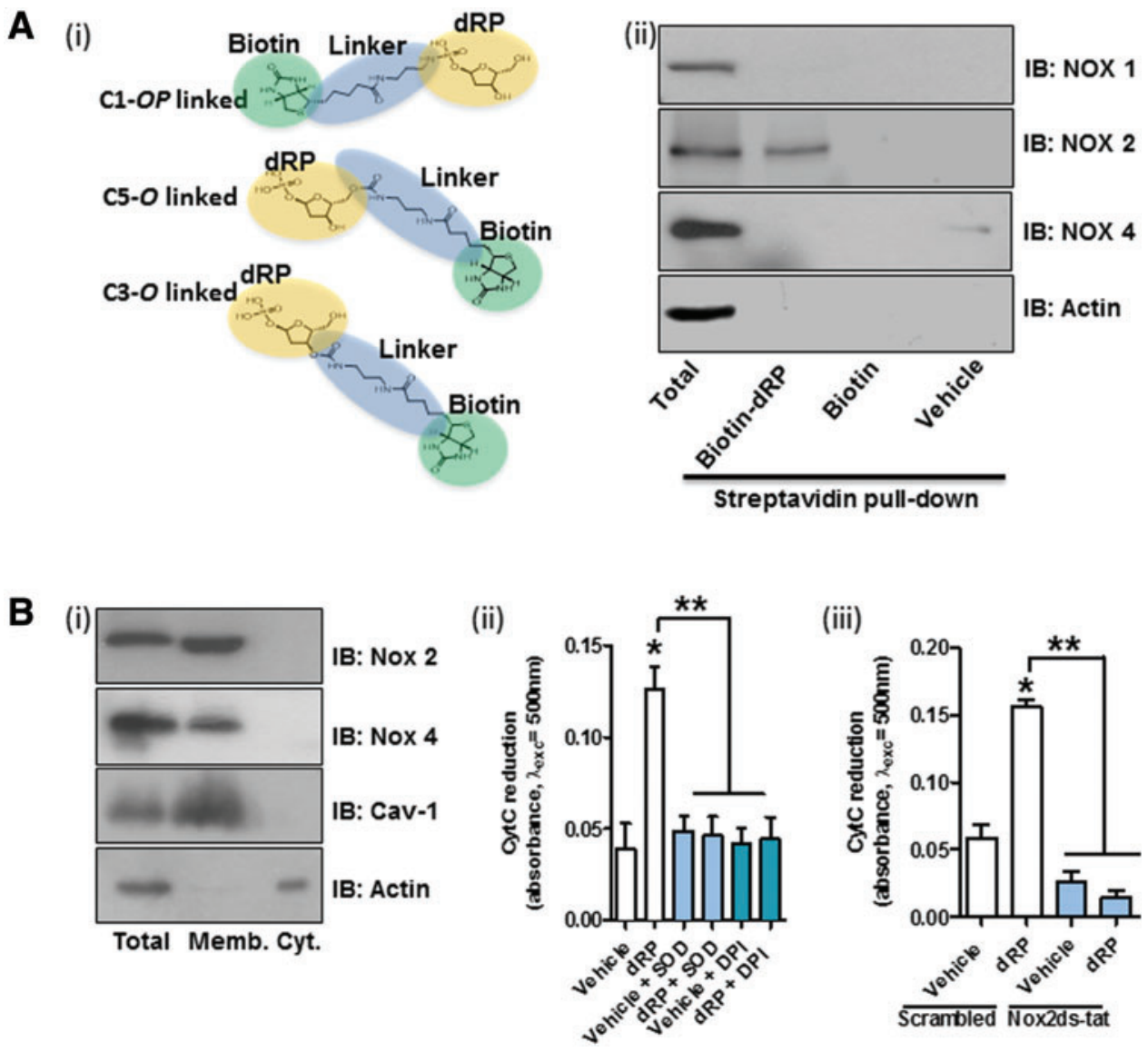

FIG. 5. dRP directly activates NOX2. (A) Binding of NOX2 was assessed by pull-down using a mixture of three dRPbiotin conjugates (i). NOX 1, 2, 4, and actin were detected by immunoblotting (ii) (four independent experiments). (B) Membrane fractions treated with dRP display NOX activation. NOX-1, 2, 4, caveolin-1, and $\beta$-actin were tested by immunoblotting (i). The membrane fractions were treated with $200 \mu M$ dRP using cytochrome c reduction assay. Cytochrome c reduction was measured as absorbance at wavelength $550 \mathrm{~nm}$. One thousand units per milliliter SOD was utilized to determine the superoxide anion-dependent component, while DPI (100 $\mu M)$ was used to determine the role of NOXs and other flavoenzymes (ii). Ten micromolars of Nox2ds-tat (or scrambled peptide as a negative control) was utilized to assess the role of NOX2 in the oxidative response measured by this membrane assay (iii). Data in B were analyzed by one-way ANOVA with Bonferroni post-test $\left[{ }^{*} p<0.05\right.$, compared with vehicle (ii) or scrambled siRNA/vehicle (iii), $* * p<0.05$ compared with dRP (ii) or scrambled siRNA/dRP (iii), $n=6$ ]. DPI, diphenylene iodonium; SOD, superoxide dismutase. To see this illustration in color, the reader is referred to the web version of this article at www.liebertpub.com/ars

FIG. 6. dRP induces oxidative stress without significantly increasing apoptosis. (A) HUVECs treated with or without $200 \mu M$ dRP for $4 \mathrm{~h}$ were labeled for $2 \mathrm{~h}$ with $20 \mu M$ BIAM in anoxic conditions. Thiol oxidation status was determined by protein separation using SDS-PAGE and staining with HRP-streptavidin. Green arrows indicate thiol oxidation, while red arrows represent thiol reduction. $\beta$-Actin immunoblotting was used to confirm equal loading. Blots are representative of four independent experiments. (B) VitaBright-43 ${ }^{\mathrm{TM}}$ staining was also utilized to measure the level of intracellular reduced thiols. HUVECs were treated with vehicle solution (Tyrode's HEPES buffer) or stimuli with/without NOX inhibitor (200 $\mu M \mathrm{dRP}, 10 \mu M \mathrm{DPI})$ for $30 \mathrm{~min}$. Cells were costained with VitaBright-43 and propidium iodide and analyzed by image cytometry using the NucleoCounter NC-3000 ${ }^{\circledR}$ system. Plots comparing VitaBright-43 (VB) intensity versus propidium iodide intensity are shown (i). Intracellular thiol oxidation was quantified by counting the percent of cells with VitaBright43 staining below 10,000 rfu (ii). Statistical analysis was performed by one-way ANOVA with Bonferroni post-test $\left({ }^{*} p<0.05\right.$, compared with vehicle, $\left.n=6\right)$. (B) Cell apoptosis was measured by flow cytometry for PE-Annexin V staining. HUVECs were treated with vehicle (Tyrode's HEPES buffer), $200 \mu M \mathrm{dRP}$, or $5 \mathrm{~m} M$ diethyl maleate (positive control) for $12 \mathrm{~h}$. FSC/SSC and Annexin V staining histograms from four independent experiments are presented (i). Data analysis is also shown (ii). Data are mean \pm SEM, analyzed by one-way ANOVA with Bonferroni post-test $(* p<0.05$, compared with vehicle, $n=6$ ). BIAM, biotinyl-iodoacetamide; FSC, forward scattering; HRP, horseradish peroxidase; SDS-PAGE, sodium dodecyl sulfate-polyacrylamide gel electrophoresis; SSC, side scattering. To see this illustration in color, the reader is referred to the web version of this article at www.liebertpub.com/ars 


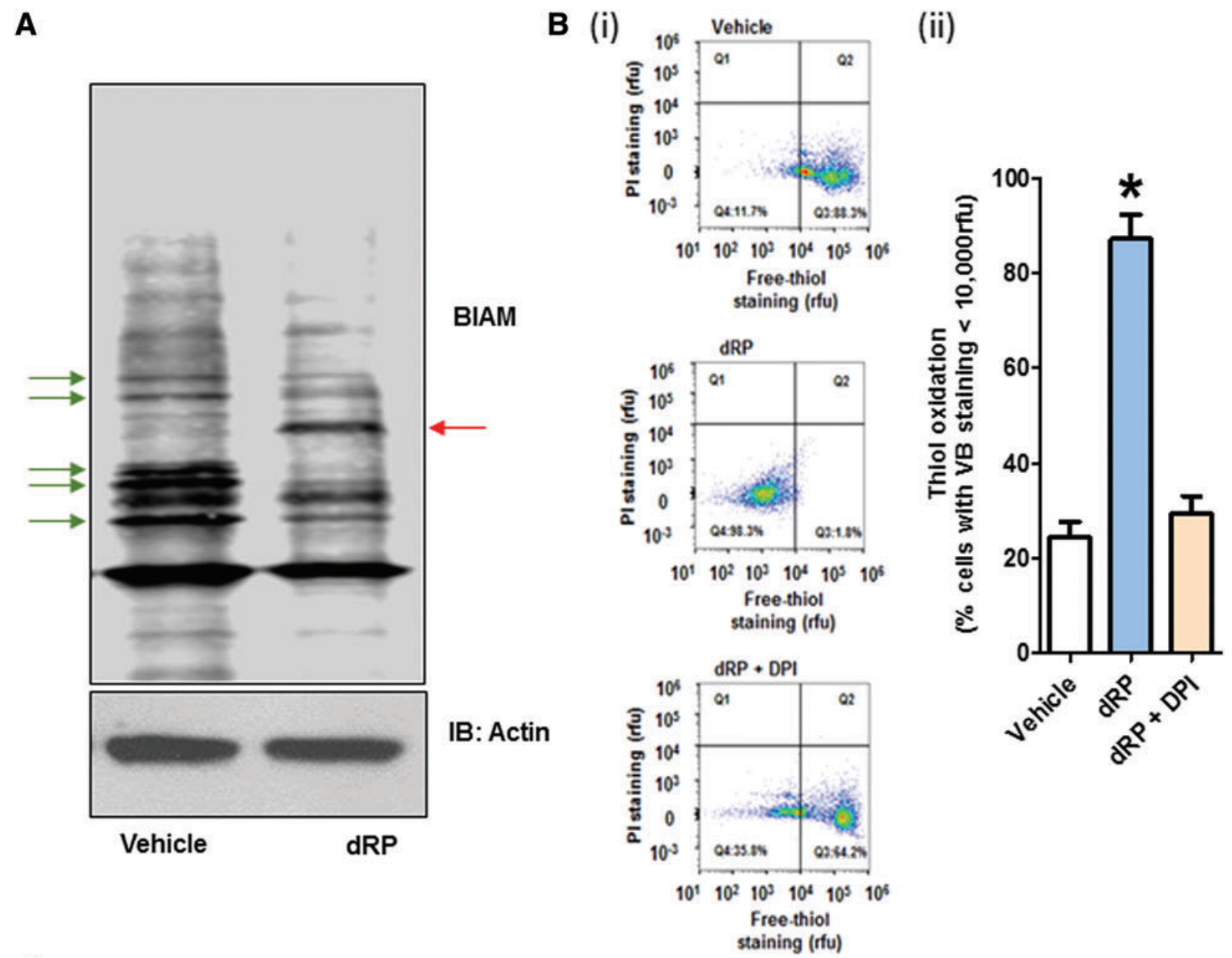

C
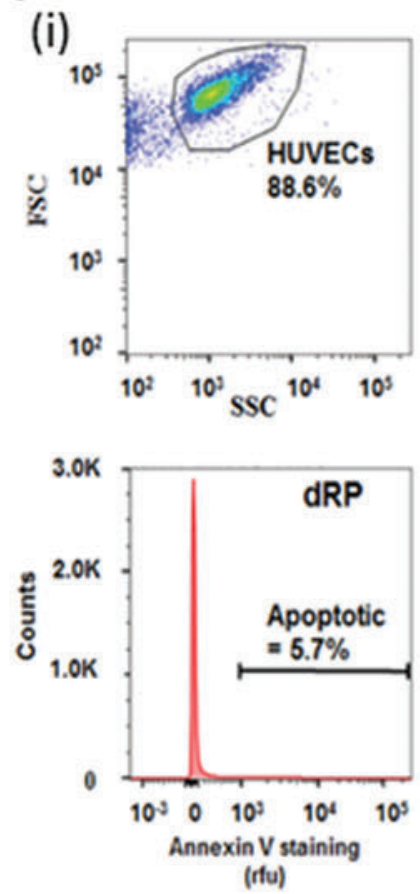
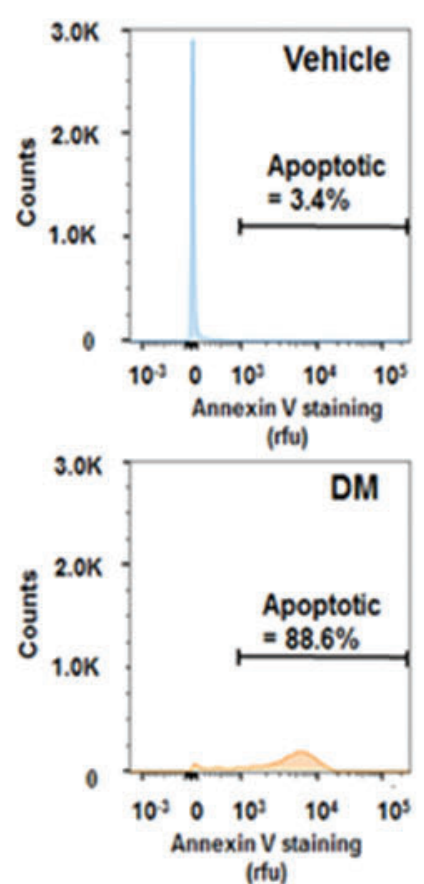

(ii)

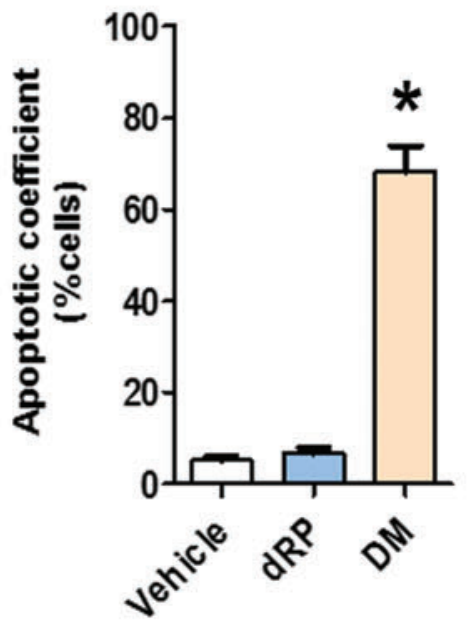



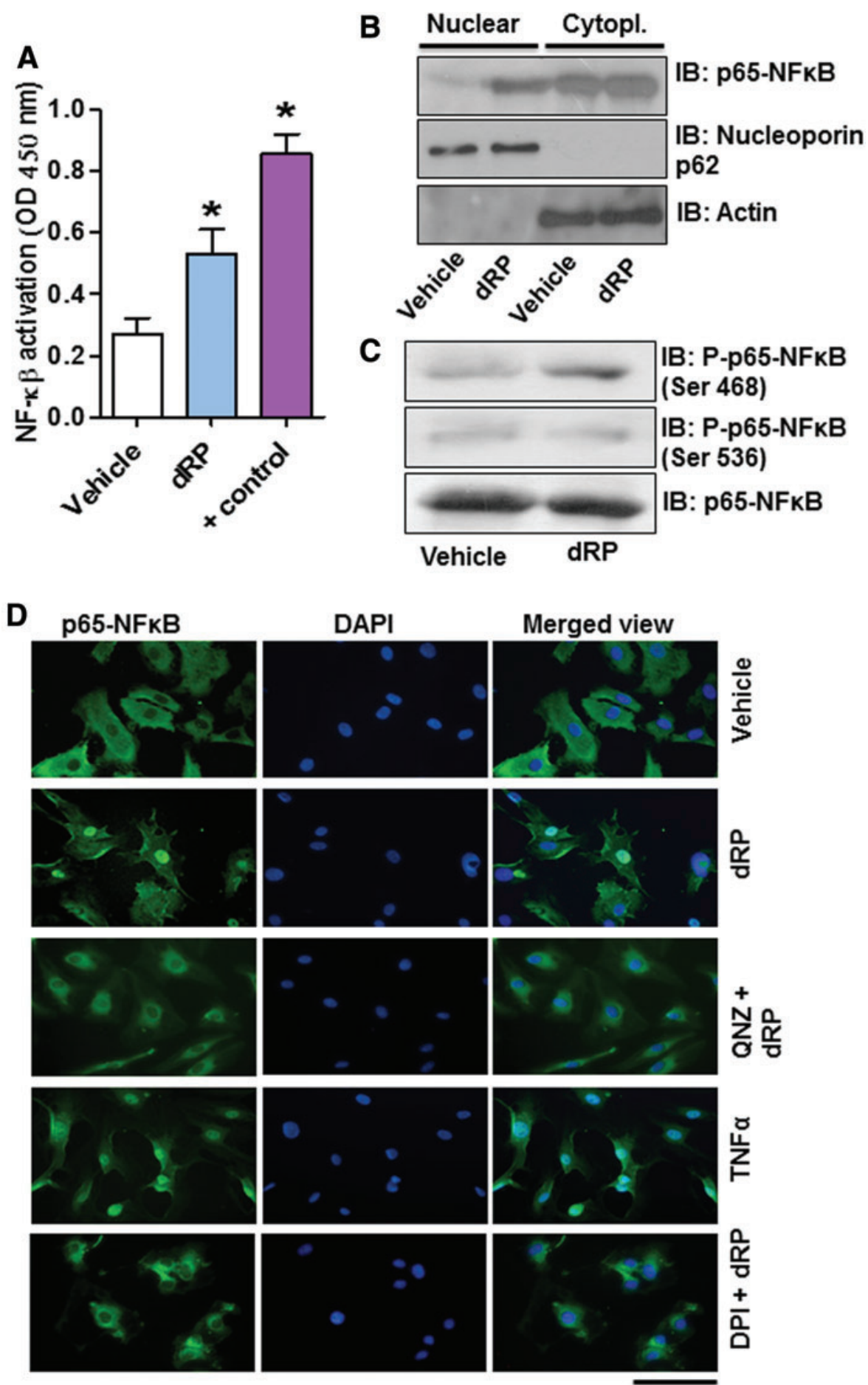

FIG. 7. NF- $\boldsymbol{\kappa} B$ is activated in response to dRP. (A) DNA binding capacity of NF- $\kappa$ B (p65 subunit) was determined using TransAM ${ }^{\circledR}$ for NF- $\kappa$ B (cat. No. 43296; Active Motif). HUVECs were treated with or without $200 \mu M$ dRP for 30 min. Whole cell extract from Jurkat cells stimulated with 12- $O$-tetradecanoylphorbol-13-acetate and calcium was used as positive control. One-way ANOVA with Bonferroni post-test was used to test statistical significance (mean \pm SEM, $n=6, * p<0.05$ ). (B) The translocation of p65-NF- $\kappa$ B to the nucleus was tested by subcellular fractionation using the NE-PER kit (Pierce). Cytoplasmic and nuclear fractions were immunoblotted for $\mathrm{p} 65-\mathrm{NF}-\kappa \mathrm{B}$, nucleoporing-p62 (a nuclear marker), and $\beta$-actin (a cytoplasmic marker). Data are representative of four independent experiments. (C) NF- $\kappa$ B activation was also investigated by phospho-specific immunoblotting. Total cell lysate was immunoblotted with phospho-specific NF- $\kappa$ B antibodies (Ser468 or Ser536) and total NF- $\kappa \mathrm{B}$ antibody (as loading control). Immunoblots represent 4 independent experiments. (D) Immunolocalization of $\mathrm{p} 65-\mathrm{NF}-\kappa \mathrm{B}$ (green) in dRP-stimulated HUVECs $(200 \mu M, 1 \mathrm{~h})$ was also tested. Where indicated, $100 \mathrm{n} M$ QNZ, an inhibitor of NF- $\kappa \mathrm{B}$ activation, was added. One hundred micromolars of DPI was used as the NOX inhibitor, whereas $50 \mathrm{ng} / \mathrm{ml} \mathrm{TNF}-\alpha$ was utilized as a positive control. Images are representative of five independent experiments. Bar: (D) $100 \mu \mathrm{m}$. NF- $\kappa$ B, nuclear factor kappa B; QNZ, N4-[2-(4-phenoxyphenyl)ethyl]-4,6-quinazolinediamine. To see this illustration in color, the reader is referred to the web version of this article at www.liebertpub.com/ars 
pro-oxidative cell conditions, leading to oxidation of cysteines in several proteins (as expected from the activation of a highly expressed pro-oxidative enzyme such as NOX2). Protein thiol oxidation (Fig. 6B) without induction of cell apoptosis (Fig. 6C) was confirmed by free thiol staining and Annexin $\mathrm{V}$ binding, respectively. $d R P$ induces activation of $N F-\kappa B$

in an NOX-dependent manner

Several redox-dependent transcription factors, including HIF-1 (51), Nrf2 $(30,34)$, and NF- $\kappa \mathrm{B}(11,56)$, play an important role in endothelial cell responses. To probe the
A
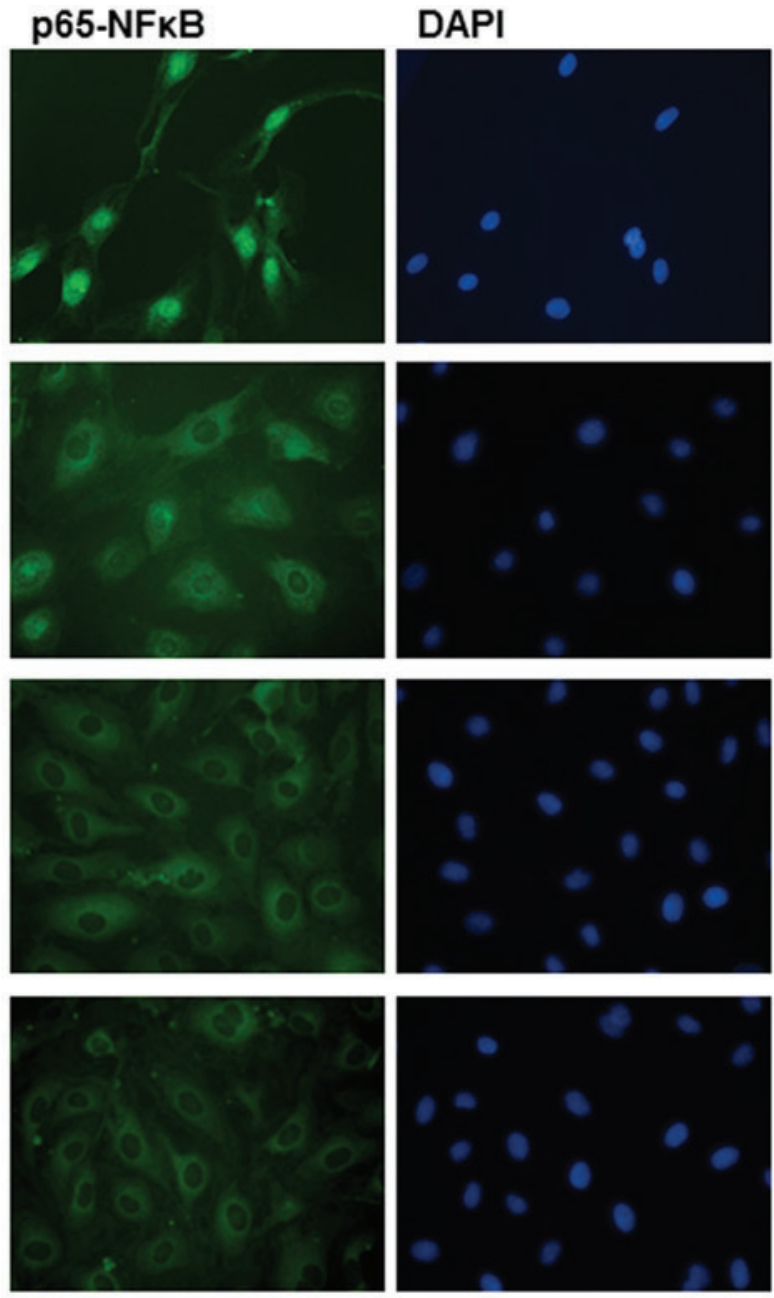

B
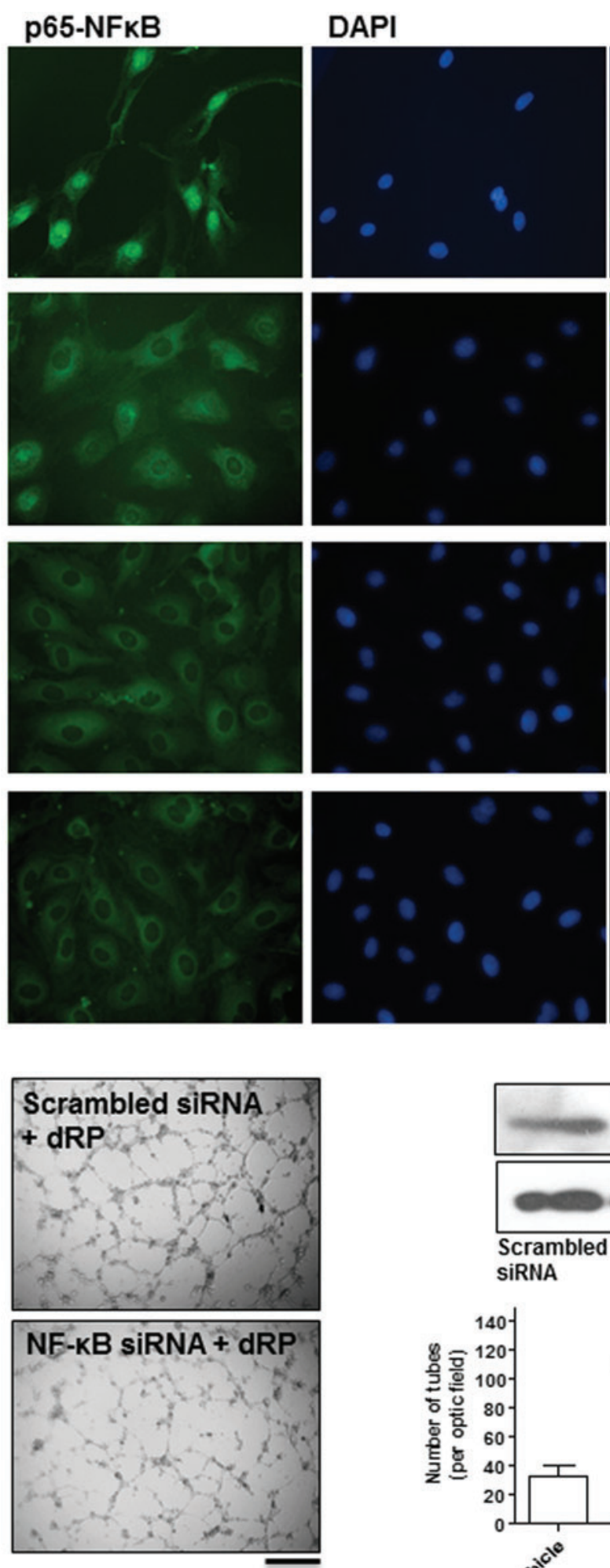

Merged view
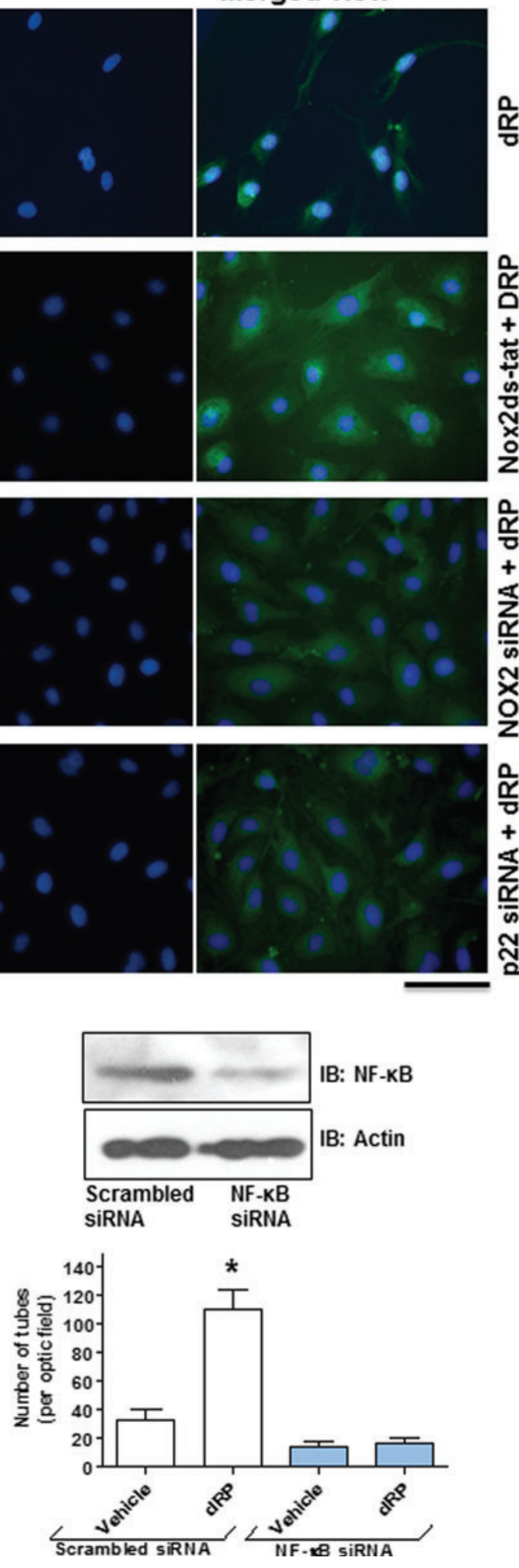

$\frac{0}{\frac{0}{\sigma}}$

FIG. 8. dRP induces NF$\kappa B$ activation in NOXdependent manner and $\mathrm{NF}-\boldsymbol{\kappa} \mathrm{B}$ activity is critical for endothelial tube formation in response to dRP. (A) Representative immunofluorescence images of HUVECs stained for NF- $\kappa \mathrm{B}$ (green) and DAPI (blue). Where indicated, HUVECs were pretreated with $10 \mu M$ Nox2ds-tat peptide for $1 \mathrm{~h}$ or subjected to NOX2 and p22phox siRNA-mediated genetic silencing for $72 \mathrm{~h}$ before treatment with $200 \mu M$ $\mathrm{dRP}$ for $30 \mathrm{~min}$. Images are representative of four independent experiments. (B) Tube formation was assessed by seeding scrambled and NF- $\kappa$ B siRNA-treated cells onto growth factor-reduced Matrigel with or without $200 \mu M$ dRP. Representative images show tube formation after $4 \mathrm{~h}$ (left) and p65-NF$\kappa \mathrm{B}$ downregulation (right). Bar graphs (bottom right) represent quantification of tube number per optical field using ImageJ software with Angiogenesis Analyzer plugin $\left({ }^{*} p<0.05\right.$, one-way ANOVA with Bonferroni post-test, $n=5$ ). Bar: (A) $100 \mu \mathrm{m}$; (B) $300 \mu \mathrm{m}$. DAPI, 4',6-diamidino2-phenylindole. To see this illustration in color, the reader is referred to the web version of this article at www .liebertpub.com/ars 
A
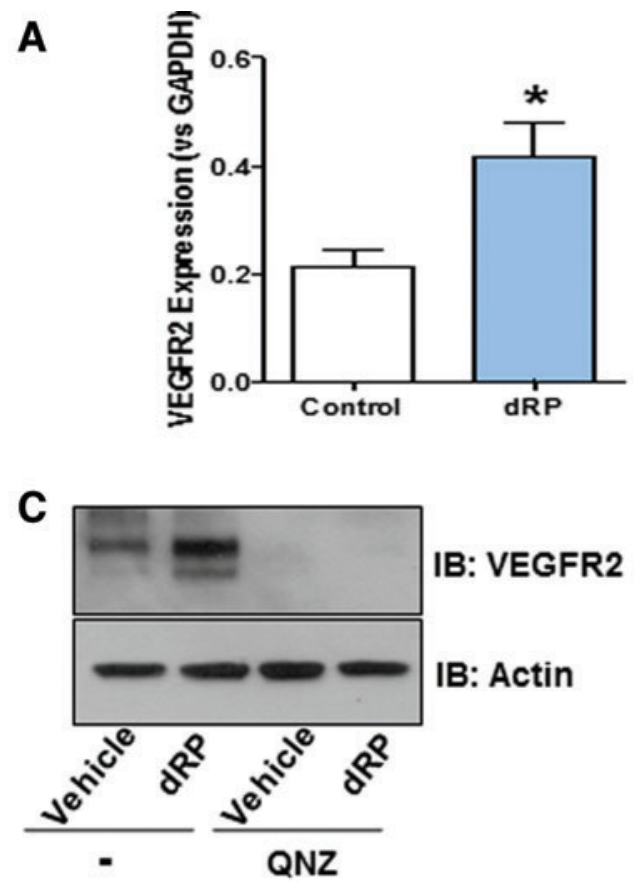

$E_{\text {(i) }}$
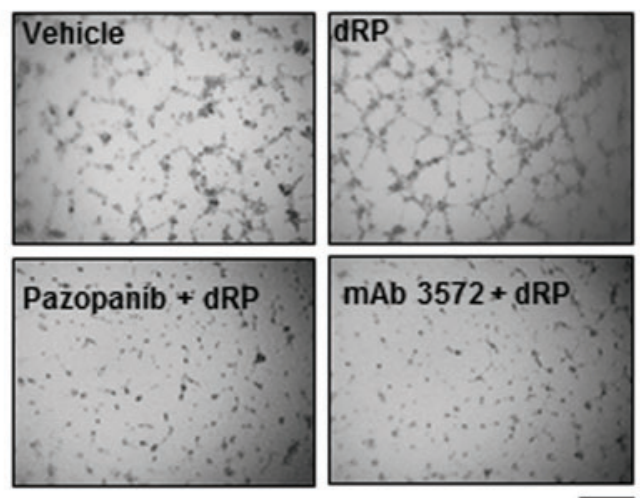

$F_{\text {(i) }}$

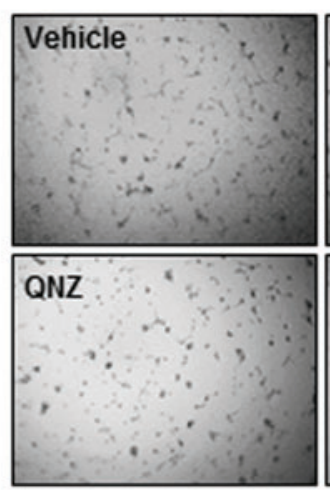

IB: VEGFR2

IB: Actin

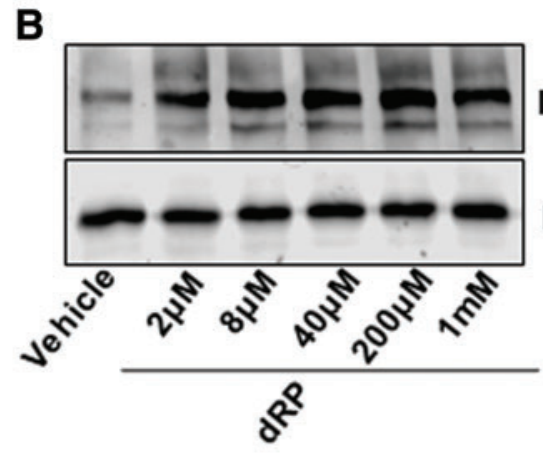

D

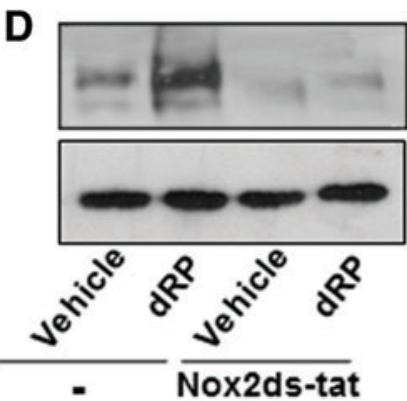

IB: VEGFR2

IB: Actin
IB: VEGFR2

IB: Actin

(ii)

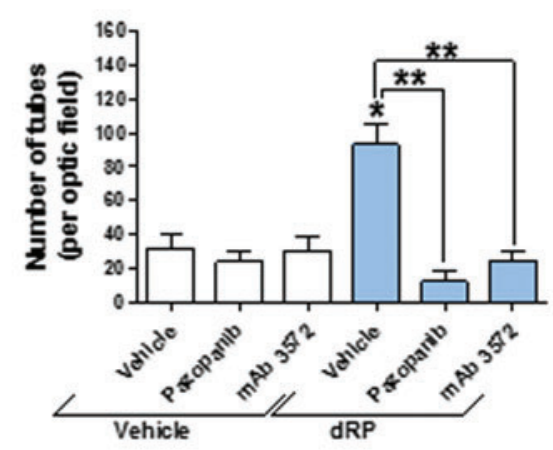

(ii)

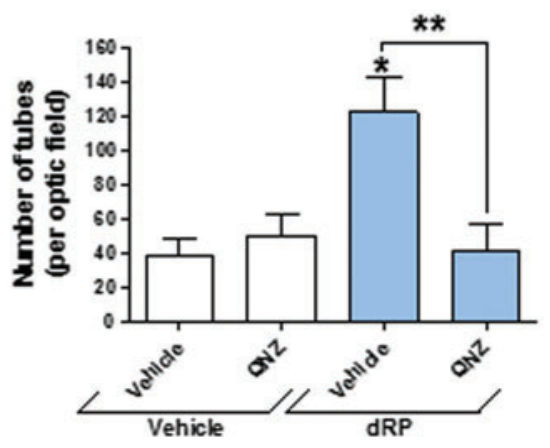

FIG. 9. VEGFR2 is upregulated in response to dRP in an NF- $\kappa \mathrm{B}$-dependent manner and its activity is necessary for dRPdependent angiogenesis. (A) qPCR analysis of VEGFR2 expression on HUVECs treated with or without $200 \mu M \mathrm{dRP}$ for $4 \mathrm{~h}$. The $2^{-4 \Lambda \mathrm{Ct}}$ analysis method was used to analyze the data with GAPDH used as normalizer. Statistical significance of the difference was tested using a nonparametric Mann-Whitney test (mean \pm SEM, $n=4,{ }^{*} p<0.05$ ). (B) HUVECs were treated with increasing concentration of dRP $(2 \mu M$ to $1 \mathrm{~m} M)$ for $6 \mathrm{~h}$. Alternatively, HUVECs were incubated with (C) $100 \mathrm{n} M$ QNZ or (D) $10 \mu M$ Nox2dstat for $1 \mathrm{~h}$ and then stimulated with $200 \mu M$ dRP for $6 \mathrm{~h}$. Cell lysates were immunoblotted for VEGFR2 and $\beta$-actin. Data are representative of four independent experiments. (E) Effects of VEGFR2 inhibitors pazopanib and mAB3572 on dRP-induced tube formation. HUVECs with or without $200 \mu M$ dRP were tested in the presence of $10 \mu \mathrm{g} / \mathrm{ml}$ pazopanib and $50 \mathrm{ng} / \mathrm{ml} \mathrm{mAB} 3572$ antibody. Representative pictures (i) and quantification (ii). (F) Effects of the NF- $\kappa$ B inhibitor QNZ (100 nM) on dRP-induced tube formation. Representative pictures (i) and quantification (ii). Bar graphs represent quantification of tube number per optical field performed using ImageJ software with Angiogenesis Analyzer plug-in and compared by one-way ANOVA with Bonferroni post-test $\left({ }^{*} p<0.05,{ }^{* *} p<0.01, n=6\right)$. Bar: (E, F) $300 \mu \mathrm{m}$. qPCR, real-time quantitative polymerase chain reaction; VEGFR2, VEGF receptor 2. To see this illustration in color, the reader is referred to the web version of this article at www.liebertpub.com/ars 
involvement of HIF-1, Nrf2, and NF- $\kappa \mathrm{B}$ as mediators of the proangiogenic activities of dRP, we tested the DNA binding activity of these transcription factors in HUVECs treated with $\mathrm{dRP}(53)$. These experiments revealed that NF- $\kappa \mathrm{B}$ is strongly activated in the presence of dRP (Fig. 7A), whereas HIF-1 and Nrf-2 did not show any significant activation (Supplementary Fig. S6B). In agreement with these results, dRP increased p65-NF- $\kappa \mathrm{B}$ levels in the nuclear fraction of HUVECs (as a result of nuclear translocation; Fig. 7B) and promoted NF- $\kappa$ B phosphorylation on Ser468 (but not Ser536; Fig. 7C and Supplementary Fig. S6A). Translocation of NF$\kappa \mathrm{B}$ to the nucleus upon $\mathrm{dRP}$ treatment was confirmed by immunolabeling and confocal imaging (Fig. 7D). Treatment with the nonspecific flavoenzyme inhibitor DPI or direct inhibition of NF- $\kappa \mathrm{B}$ with the potent inhibitor N4-[2-(4phenoxyphenyl)ethyl]-4,6-quinazolinediamine (QNZ) (61) abolished nuclear translocation of NF- $\kappa$ B. Similar to DPI, NOX2 inhibition by the peptide Nox2ds-tat and genetic silencing of p22phox or NOX2 abolished translocation of NF$\kappa \mathrm{B}$ to the nucleus in dRP-stimulated HUVECs (Fig. 8A). Collectively, these data suggest that dRP-dependent activation of NOX2 is responsible for $\mathrm{NF}-\kappa \mathrm{B}$ activation. The functional role of NF- $\kappa \mathrm{B}$ in the proangiogenic activity of dRP was tested by siRNA-dependent genetic silencing of this transcription factor (Fig. 8B), which resulted in complete inhibition of endothelial tube formation in response to dRP.

\section{dRP-dependent activation of $N F-\kappa B$ upregulates} VEGFR2 and induces endothelial tube formation

An initial analysis of the expression of angiogenic factors in HUVECs by enzyme-linked immunosorbent assay (ELISA) (Supplementary Fig. S7A) and immunoblotting (Supplementary Fig. S7B) did not detect any significant change in response to $d R P$, suggesting that the effects of $d R P$ do not depend upon autocrine production of these factors. Further studies, however, showed that VEGFR2 is robustly upregulated at the messenger RNA (mRNA) and protein levels in HUVECs following exposure to dRP (Fig. 9A-D). The NF$\kappa \mathrm{B}$ inhibitor QNZ ablated the expression of VEGFR2 in HUVECs and inhibited the upregulation of this receptor by $\mathrm{dRP}$, suggesting a critical role for NF- $\kappa \mathrm{B}$ in the expression of VEGFR2 (Fig. 9C). The NOX2 inhibitor Nox2ds-tat
(54) significantly reduced upregulation of VEGFR2 by dRP (Fig 9D). The functional relevance of VEGFR2 upregulation for the proangiogenic activity of dRP was confirmed in experiments using the VEGFR2 inhibitor pazopanib and the VEGFR2-specific inhibitory antibody MAB3572, both of which abolished tubulogenesis stimulated by dRP (Fig. 9E). Experiments with the NF- $\kappa \mathrm{B}$ inhibitor QNZ suggested that this transcription factor is necessary for dRP-dependent tube formation (Fig. 9F), which correlates with the strong effect of $\mathrm{NF}-\kappa \mathrm{B}$ inhibition on VEGFR2 expression shown in Figure 9B, and the involvement of VEGFR2 in dRP-dependent angiogenesis shown in Figure 9E.

\section{dRP stimulates VEGFR2 upregulation and NOX2-dependent angiogenesis in vivo}

The proangiogenic activity of dRP has been described previously $(35,38,49,50)$. To confirm that the mechanism of action of $\mathrm{dRP}$ that we characterized in vitro also occurs in vivo, we applied dRP to excisional wounds in mice and assessed the levels of tissue vascularization by hematoxylin staining (Fig. 10A) and VEGFR2 expression at the wound site 7 days after application (Fig. 10B). These experiments showed a significant increase in both wound vascularity and expression of VEGFR2 in wounds treated with dRP. The dependence of dRP proangiogenic activity on NOX2 was then tested in $\mathrm{NOX}_{2}{ }^{-/-}$mice. Using a Matrigel plug vascularization assay, we demonstrated that the presence of dRP stimulated a significantly higher vascularization of the plug in wild-type animals compared with $\mathrm{NOX}^{-/-}$mice. This was demonstrated by hematoxylin (Fig. 10C) and endothelialspecific CD31 staining (Fig. 10D).

\section{Discussion}

The proangiogenic activity of dRP has been described previously, although the mechanism of action has remained elusive. The dRP-generating enzyme TP was initially cloned as platelet-derived endothelial cell growth factor (PD-ECGF) and characterized for its proangiogenic properties (27). Local injection of TP or TP-expressing cells has been shown to induce tissue neovascularization in vivo, which depends on the enzymatic activity of TP and generation of dRP (35). TP

FIG. 10. dRP stimulates VEGFR2 upregulation and NOX2-deoendent angiogenesis in vivo. (A) Representative examples (i) and quantification (ii) of the hematoxylin/eosin staining of wound tissue treated with dRP or vehicle control (PBS). Data represent counts of vascular structures (surrounded by continuous intimal monolayer) per optic field. Statistical significance of the difference was assessed by nonparametric Mann-Whitney test $(* p<0.05$ compared with vehicle, $n=10)$. Vascular structures are highlighted by white arrows in the picture. (B) Representative examples (i) and quantification (ii) of the VEGFR2-specific staining of wound tissue treated with dRP or vehicle control (PBS). Data represent counts of high fluorescence intensity areas per $\mathrm{mm}^{2}$, as estimated using ImageJ. Statistical significance of the difference was assessed by nonparametric Mann-Whitney test $(* p<0.05$ compared with vehicle, $n=8)$. (C) Representative examples (i) and quantification (ii) of hematoxylin staining of Matrigel plugs containing dRP (41.2 $\mu \mathrm{g} / \mathrm{plug})$ or vehicle control (PBS) after $7 \mathrm{days}$ of implantation in wild-type (C57BL6/J) or NOX2 $2^{-/-}$(B6.129S-Cybbtm1Din/J; Jackson Laboratories) mice. Data represent counts of capillary structures per $\mathrm{mm}^{2}$, as estimated using ImageJ. Statistical significance of the difference was assessed by one-way ANOVA with Bonferroni post hoc test $(* p<0.05$ compared with vehicle, $* * p<0.05$ compared with $\mathrm{dRP}, n=6)$. (D) Representative examples (i) and quantification (ii) of the DAPI/CD31-specific staining of Matrigel plugs containing dRP $(41.2 \mu \mathrm{g} / \mathrm{plug})$ or vehicle control (PBS) after 7 days of implantation in wild-type (C57BL6/J) or NOX2 ${ }^{-/-}$(B6.129SCybbtm1Din/J; Jackson Laboratories) mice. Data represent counts of capillary structures per mm ${ }^{2}$, as estimated using ImageJ. Statistical significance of the difference was assessed by one-way ANOVA with Bonferroni post hoc test $(* p<0.05$ compared with vehicle, $* * p<0.05$ compared with dRP, $n=6$ ). Bars: $300 \mu \mathrm{m}$ (throughout). To see this illustration in color, the reader is referred to the web version of this article at www.liebertpub.com/ars 
and other dRP-generating enzymes, such as PNP and UP, are overexpressed in cancers and associated with cancer vascularization and metastasis (31). In our studies, we identified platelets and macrophages as key generators of micromolar concentrations of dRP (49), whereas endothelial cells do not synthesize detectable levels of dRP and recognize this molecule (or its dephosphorylation product 2-deoxy-D-ribose) as a paracrine signal that triggers angiogenesis (6).
In this study, we demonstrate for the first time that the oxidative response induced by $\mathrm{dRP}$ in endothelial cells is mediated by NOX 2 and that $\mathrm{dRP}$ can directly bind and activate this enzyme. Importantly, we showed that dRP binds and pulls down NOX2, but not NOX1 or NOX4, from endothelial cell lysates. As NOX1 and NOX2 complexes share several regulatory subunits (i.e., p22phox, p47phox, and Rac1), the specific pull-down of NOX2, and not NOX1, by biotinylated
$\mathbf{A}$ (i)

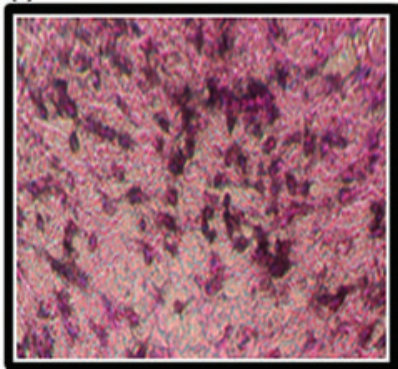

Vehicle

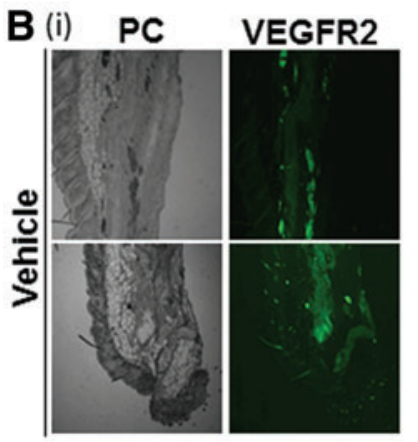

C (i)
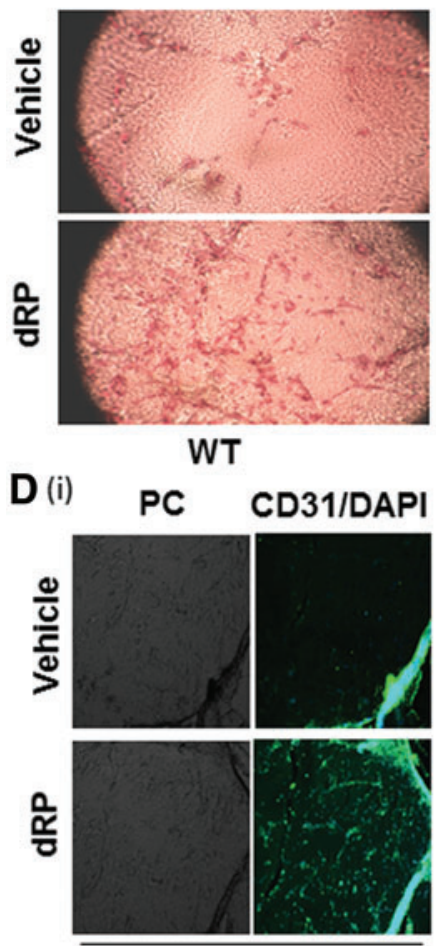

WT

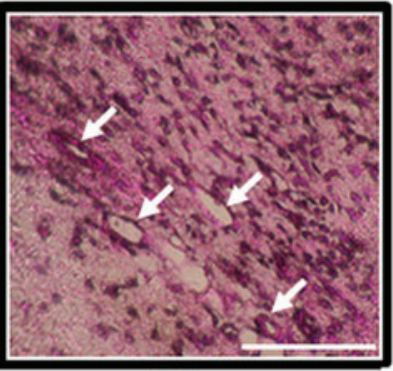

dRP

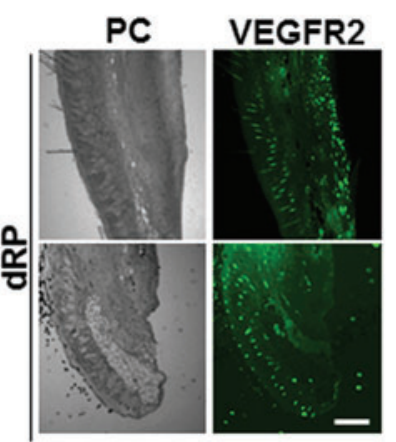

(ii)
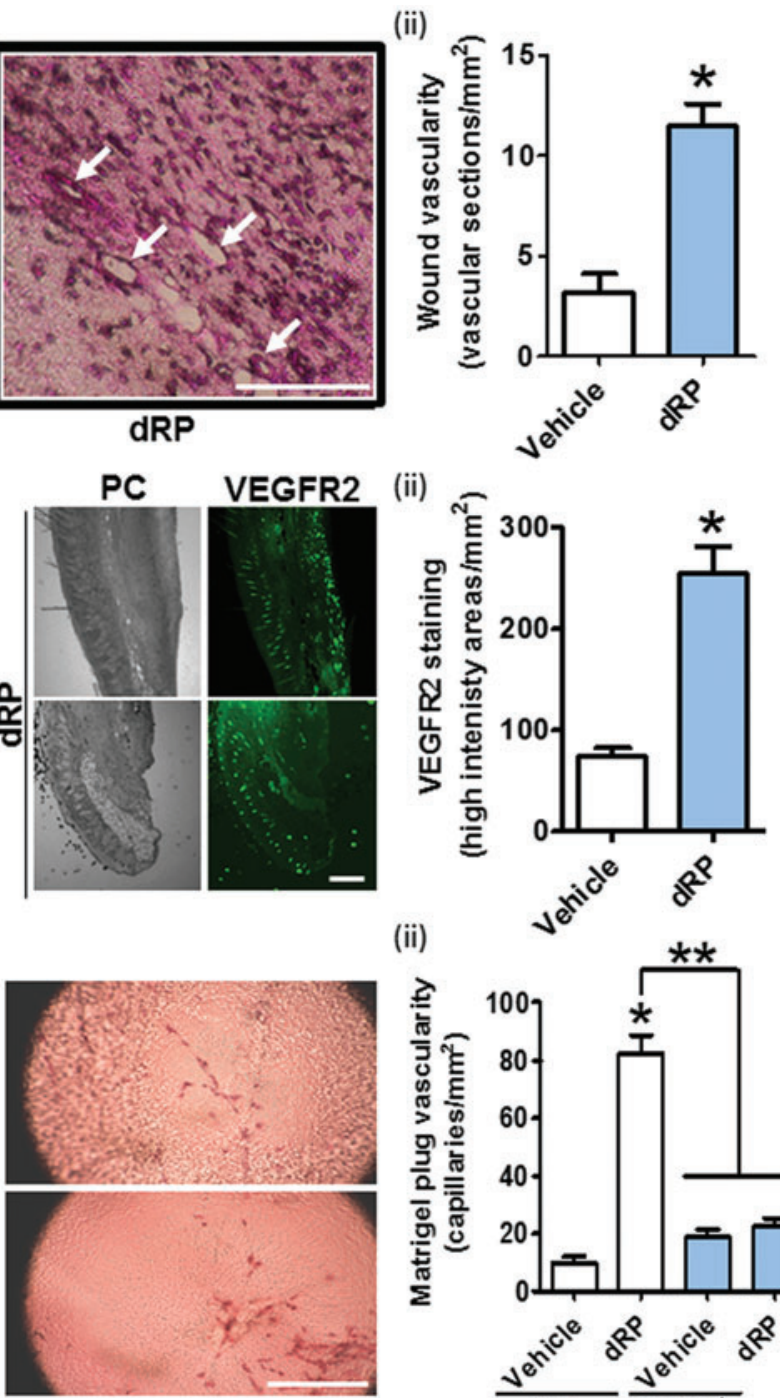

NOX2-

PC CD31/DAPI
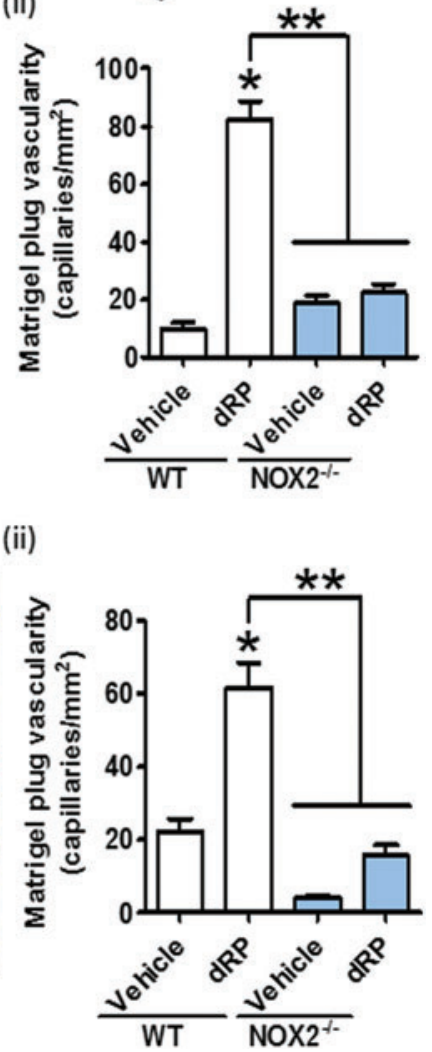
dRP supports the hypothesis that dRP directly interacts with the NOX2 enzymatic subunit (also known as gp91phox). Direct binding and activation of the NOX complex are remarkable and rarely reported modes of action for a signaling molecule, which have been described in only a few recent studies. Arachidonic acid has been shown to activate NOX2 by promoting its interaction with the p67phox/Rac1 complex (37). In this case, the authors proposed that arachidonic acid interacts directly with p67phox. The involvement of p47phox, p67phox, or other subunits in the dRP-dependent activation of NOX2 demonstrated in our study cannot be excluded at present, but the initial binding appears to be to NOX2.

A correlation between NOX-dependent ROS generation and angiogenesis has been described previously. NOX4 and NOX2 both play prominent roles in the stimulation of angiogenic responses of endothelial cells $(15,39,40,63,69)$, whereas NOX1 and NOX5 have received less attention $(3,4)$. In particular, a link between NOX2 and NOX4 activity and increased endothelial cell motility has been reported $(26,45)$. In our study, NOX 2 activation by dRP leads to NF- $\kappa \mathrm{B}$ activation (as shown by several approaches, including p65$\mathrm{NF}-\kappa \mathrm{B}$ translocation and phosphorylation), which appears critical in the proangiogenic signaling cascade triggered by $\mathrm{dRP}$. The role of NF- $\kappa \mathrm{B}$ in the signaling cascade stimulated by dRP was demonstrated using both the QNZ inhibitor [which not only has low nanomolar potency on NF- $\kappa \mathrm{B}$ but can also inhibit store-operated calcium channels at high nanomolar concentrations (68)] and genetic silencing of p65$\mathrm{NF}-\kappa \mathrm{B}$. This is in agreement with recent studies showing activation of $\mathrm{NF}-\kappa \mathrm{B}$ downstream of NOX2 in angiogenic responses of endothelial cells (40). However, in contrast to this latter study, we did not detect any significant changes in the expression of VEGF-A or angiopoietin- 2 in response to $\mathrm{dRP}$ treatment. One possible explanation for these contrasting findings is the source of endothelial cells used in the two studies (i.e., human umbilical vein vs. human pulmonary microvasculature). Instead, we identified VEGFR2 as a key component of the $\mathrm{NF}-\kappa \mathrm{B}$-mediated angiogenic response stimulated by dRP. This is in keeping with previous studies showing that the promoter of VEGFR2 contains NF- $\kappa \mathrm{B}$ binding motives (55) and recent studies showing regulation of VEGFR2 expression by NF- $\kappa \mathrm{B}$ (17). Importantly, we confirmed VEGFR2 upregulation by dRP in vivo using a mouse excisional wound assay.

Several previous studies have highlighted associations between endothelial cell oxidative status and the angiogenic response. For example, the link between ROS formation in hypoxic conditions and the activity of HIF-1 has been described (22). Although additional routes have also been proposed (2), ROS have been shown to promote HIF-1dependent transcription via inactivation of prolyl hydroxylase and reduction of HIF proteasome-dependent degradation (47). The activation of NOX4 and NOX3, but not NOX2, has previously been associated with HIF-1 activation $(8,69)$, whereas NOX2 has been shown to stimulate NF- $\kappa \mathrm{B}$ activation in endothelial cells (40). Since we have identified NOX2 as a key target of dRP biological activity, our data support the existing literature showing that $\mathrm{NF}-\kappa \mathrm{B}$, as opposed to HIF-1, is activated by this member of the NOX family. We confirmed that NOX2 is also necessary for the proangiogenic effect of dRP in vivo. This is in line with two recent studies showing a role for this member of the NOX family in the stimulation of angiogenesis in vivo, although it should be emphasized that these reports, in contrast to the present study, did not provide clarity on the underlying molecular mechanisms $(10,70)$.

Interestingly, we observed direct cysteine oxidation in endothelial cells treated with $\mathrm{dRP}$ (as demonstrated by the loss of free thiols). The product of cysteine oxidation by ROS in mammalian cells is cysteine sulfenic acid, which can undergo further oxidation to generate cysteine sulfinic acid and cysteine sulfonic acid or can form intramolecular or intermolecular disulfide bridges (16). The oxidation of cysteine by ROS has important functional consequences for protein phosphatases and protein kinases (52). For example, and relevant to our study, ROS-induced serine and/or tyrosine phosphorylation, ubiquitination, and consequent proteolytic degradation of the inhibitor subunit, inhibitor of NF- $\kappa \mathrm{B}(\mathrm{I} \kappa \mathrm{B})$, are responsible for nuclear transportation and activation of NF- $\kappa \mathrm{B}$ (43). These observations support our data showing ROS-dependent activation of $\mathrm{NF}-\kappa \mathrm{B}$ in response to $\mathrm{dRP}$.

Overall, this study has comprehensively elucidated the mechanism of action of dRP, (Supplementary Fig. S8) a small molecule with proangiogenic effects in vitro and in vivo (49). We showed that similarly to other sugars (either hexoses or pentoses) $(29,44)$, dRP is readily internalized by endothelial cells and that the transporter GLUT1 is the most likely transporter responsible for this. This is in agreement with previous studies suggesting that TP stimulates endothelial cells in a nonreceptor-mediated manner (6). Once internalized, dRP has the remarkable ability to directly bind and activate NOX2, but not NOX4 or NOX1. Activation of NOX2 and the resulting generation of ROS trigger NF- $\kappa \mathrm{B}$ activation and promote angiogenic responses in endothelial cells, which depend on the activity of VEGFR2. This study, therefore, represents a milestone in the understanding of the proangiogenic activity of the dRP-generating enzyme TP, which was cloned as platelet-derived endothelial growth factor and characterized as a potent stimulator of angiogenesis in the late 1980s (27).

\section{Materials and Methods}

\section{$L C-M S$ detection and quantification of $d R P$}

Macrophages were differentiated from whole bone marrow cultures from B57BL6 mice in Dulbecco's modified Eagle's medium $+10 \%$ L929 cell-conditioned medium, 10\% fetal bovine serum (FBS), and $1 \times$ penicillin/streptomycin. Activation of macrophages was performed with $100 \mathrm{ng} / \mathrm{ml}$ LPS and $100 \mathrm{ng} / \mathrm{ml}$ interferon gamma (IFN $\gamma$ ) or $20 \mathrm{ng} / \mathrm{ml}$ interleukin 4 (IL4) and $50 \mathrm{ng} / \mathrm{ml}$ IFN $\gamma$. Platelets were isolated via two-step centrifugation, as previously described (66). Activation was obtained with $0.1 \mathrm{U} / \mathrm{ml}$ thrombin or $10 \mu \mathrm{g} / \mathrm{ml}$ collagen for $10 \mathrm{~min}$ in an aggregometer at $700 \mathrm{rpm}$. Endothelial cells with or without dRP incubation $(200 \mu M)$ were washed with phosphate-buffered saline (PBS) three times and lysed by ultrasonication. For all samples (i.e., cell extracts obtained by ultrasonication or culture supernatants), proteins were eliminated by acetonitrile precipitation. Quadrupole time-of-flight (QTOF)-UHPLC analysis was conducted using a MaXis HD electrospray ionization (ESI)-QTOF mass spectrometer (Bruker Daltonik GmbH, Bremen, Germany), which was coupled to an Ultimate 3000 UHPLC (Thermo Fisher Scientific). LC was performed using a Dionex 
Acclaim RSLC PolarAdvantage II (PA2), $2.2 \mu M, 120 \AA$, $2.1 \times 50 \mathrm{~mm}$ reverse-phase column (Thermo Fisher Scientific) with a flow rate of $0.4 \mathrm{ml} / \mathrm{min}$ and an injection volume of $10 \mu$ l. Mobile phases A and B consisted of $1 \mathrm{~m} M$ ammonium fluoride in water and methanol, respectively. dRP was detected as $[\mathrm{M}-\mathrm{H}]$ - ion with a mass-to-charge $(\mathrm{m} / \mathrm{z})$ ratio of 314.0120 $\pm 0.005 \mathrm{Da}$.

\section{Endothelial cell culture}

HUVECs were isolated from umbilical veins as described previously (25). HUVECs were cultured and passaged in medium M199 supplemented with 20\% FBS, $4 \mathrm{~m} M$ glutamine, $100 \mathrm{U} / \mathrm{ml}$ penicillin, $100 \mathrm{U} / \mathrm{ml}$ streptomycin, and $20 \mathrm{mM} \mathrm{NaH}-$ $\mathrm{CO}_{3}$ and cultured in flasks precoated with $1 \%$ gelatin $(\mathrm{w} / \mathrm{v})$.

\section{In vitro capillary-like tube formation assay}

Growth factor-reduced (GFR) Matrigel was utilized to provide extracellular matrix for cell culture; $10^{4}$ cells/well in $100 \mu \mathrm{l}$ of M199 (no FBS) were added to 96-well microplates containing $65 \mu \mathrm{l}$ GFR Matrigel per well. Phase-contrast images were captured $4 \mathrm{~h}$ after treatment using an EVOS FL microscope with a $4 x / 0.1$ Plan-Achromat objective. Total number of tubes was measured using the Angiogenesis Analyzer plug-in of ImageJ.

\section{Intracellular live-cell ROS generation assay}

HUVECs were plated into 96-well, black, optically clear bottom, tissue culture sterile plates and were cultured for $24 \mathrm{~h}$ to reach $\sim 70 \%$ confluence. Cells were washed once with PBS, pretreated with inhibitors for $30 \mathrm{~min}$ (as indicated in Figs. 1D, 3A-C, 3E, and 4C), and then treated with $5 \mu M$ DHE and dRP at the desired concentrations. Fluorescence was monitored using a CLARIOstar ${ }^{\circledR}$ plate reader at $37^{\circ} \mathrm{C}$ with atmospheric control at $5 \% \mathrm{CO}_{2} / 20 \% \mathrm{O}_{2}$. Fluorescence was measured at Ex/Em: 510/610 nm over $2 \mathrm{~h}$.

\section{Superoxide detection using EPR}

HUVECs cultured in six-well plates were washed twice with EPR-Krebs HEPES buffer (EPR-KHB) adjusted to $\mathrm{pH}$ 7.4 and then incubated for $1 \mathrm{~h}$ in deoxygenated EPR-KHB $(+1 \mathrm{~g} / \mathrm{l}$ glucose $)$ in the presence of treatments. Cell-permeable superoxide-specific spin probe $\mathrm{CMH}$ at a final concentration of $200 \mu M$ (from $10 \mathrm{mM}$ CMH stock solution in the presence of $25 \mu M$ deferoxamine and $5 \mu M$ DETC) was then added for $45 \mathrm{~min}$. The supernatant was then analyzed using a Bruker escan with the following settings: center field $1.99 \mathrm{~g}$, microwave power $20 \mathrm{~mW}$, modulation amplitude $2 \mathrm{G}$, sweep time $10 \mathrm{~s}$, number of scans 10 , and field sweep $60 \mathrm{G}$. Calibration curve was calculated using known concentrations of CM. (CM-radical, No. NOX-18.2; purchased from Noxygen), as shown in Figure 2C. Data are presented as pmols of superoxide anion divided by incubation time in minutes and amount of protein per well in $\mathrm{mg}$, calculated using the bicinchoninic assay method.

\section{Determination of NOX activity in endothelial membranes}

The membrane fraction of HUVECs was separated by ultrasonication and ultracentrifugation $(100,000 \mathrm{~g}$ for
$60 \mathrm{~min}$ ). NOX activity in the membrane fraction was measured as previously described (9). Superoxide production was measured in PBS $(140 \mathrm{~m} M \mathrm{NaCl}, 2.7 \mathrm{~m} M \mathrm{KCl}, 10 \mathrm{~m} M$ $\mathrm{Na}_{2} \mathrm{HPO}_{4}, 2 \mathrm{mM} \mathrm{KH}_{2} \mathrm{PO}_{4}$, pH 7.4) containing $100 \mu \mathrm{g}$ of the membrane fraction, $10 \mathrm{~m} M$ diethyldithiocarbamate, $100 \mu M$ $\mathrm{NADPH}, 80 \mu M$ acetylated cytochrome C, $1000 \mathrm{U} / \mathrm{ml}$ catalase, and $100 \mu M$ EDTA; $10 \mu M$ DPI and $1000 \mathrm{U} / \mathrm{ml}$ SOD were used to inhibit and scavenge ROS, respectively.

\section{ELISA-based assay for transcription factor activation}

DNA binding capacity of NF- $\kappa \mathrm{B}$ was determined in whole extracts of HUVECs treated as indicated using the Trans$\mathrm{AM}^{\circledR}$ method according to the suppliers' instructions.

\section{Immunofluorescence of $N F-\kappa B$ translocation}

HUVECs were grown on coverslips to 60-70\% confluence and treated with stimuli/inhibitors. Following fixation in $4 \%$ w/v paraformaldehyde, cells were stained with anti-p65-NF$\kappa \mathrm{B}(1: 50)$, Alexa Fluor ${ }^{\circledR} 488$ rabbit anti-mouse (1:200), and 4',6-diamidino-2-phenylindole (DAPI, 1:100). Slides were examined using the $60 \times 1.40$ NA oil objective on an LSM 510 META confocal microscope (Carl Zeiss AG, Jena, Germany).

\section{Real-time quantitative polymerase chain reaction}

Real-time quantitative polymerase chain reaction (qPCR) was performed on a ViiA7 Real-Time PCR System using Power SYBR Green PCR and $300 \mathrm{n} M$ gene-specific primers (VEGFR2: 5'CCAGTGTCATTTCCGATCACT TT and 5'GGCCCAATAATCAGAGTGGCA and GAPDH: 5'-AGC CGCATCTTCTTT TGCGT and 5'-TGACGAACATGGG GGCATCA). The amplification of a single PCR product was confirmed by melting curve analysis. Gene-specific mRNA levels were estimated by the $2^{-\Delta \Delta \mathrm{Ct}}$ analysis and normalized against GAPDH levels to obtain relative changes in gene expression.

\section{Immunoprecipitation and immunoblotting}

For immunoprecipitation, HUVECs cultured in six-well microplates were lysed in $500 \mu \mathrm{l}$ of ice-cold lysis buffer (50 $\mathrm{m} M$ Tris- $\mathrm{HCl}, \mathrm{pH} 8.0,150 \mathrm{~m} M \mathrm{NaCl}$, and $0.1 \%$ Triton $\mathrm{X}-100$ containing protease and phosphatase inhibitors). The suspension was ultrasonicated using a 150VT Ultrasonic Homogenizer (BioLogics, Manassas, VA). Primary antibody and protein A/G Plus Agarose were used for immunoprecipitation. Immunoprecipitates were subjected to sodium dodecyl sulfate-polyacrylamide gel electrophoresis (SDSPAGE) and transferred to polyvinylidene difluoride for immunoblotting. For immunoblotting, cells were lysed in RIPA buffer (1\% v/v Triton X-100, $1 \%$ w/v sodium deoxycholate, $0.1 \%$ w/v SDS, $150 \mathrm{~m} M \mathrm{NaCl}, 5 \mathrm{~m} M$ EDTA, $50 \mathrm{~m} M$ Tris, $\mathrm{pH} 7.4)$.

\section{siRNA-mediated gene silencing}

HUVECs were transfected with NOX2 (sc-35503), NOX4 (sc-41586), p65 NF- $\kappa \mathrm{B}$ (sc-29410), p22phox (sc-36149), or scrambled control (sc-37007) siRNAs (Santa Cruz Biotechnology). The siRNAs were diluted to $100 \mathrm{n} M$ in transfection medium (sc-36868; Santa Cruz Biotechnology) 
containing transfection reagent (sc-29528; Santa Cruz Biotechnology), incubated for $45 \mathrm{~min}$ at room temperature, and then further diluted to $20 \mathrm{n} M$ in transfection medium. The cells were covered with this solution and incubated for $3 \mathrm{~h}$ at $37^{\circ} \mathrm{C}$. The solution was then replaced with fresh culture medium and cells used for experiments $72 \mathrm{~h}$ after transfection.

\section{Preparation of a biotinylated $d R P$ bait and streptavidin pull-down assay}

$\mathrm{dRP}$ at $0.6 \mathrm{mg} / \mathrm{ml}$ was resuspended in anhydrous dimethyl sulfoxide (DMSO) and 1,1'-carbonyldiimidazole. The resulting solution was stirred for $24 \mathrm{~h}$. In a separate vessel, $\mathrm{N}$-(+)-biotinyl-3-aminopropylammonium trifluoroacetate was taken up in anhydrous dimethylformamide and N,Ndiisopropylethylamine added. After $30 \mathrm{~min}$, the solvents were evaporated to dryness and the DMSO solution of activated dRP was added. The resulting solution was stirred for $72 \mathrm{~h}$ before being divided into 10 equal portions and the solvents removed under vacuum. HUVECs were scraped on ice into $10 \mathrm{~m} M$ Tris plus $0.3 M$ sucrose buffer ( $\mathrm{pH} 7.1)$. The suspension was sonicated on ice and centrifuged at $1000 \mathrm{~g}$ for $5 \mathrm{~min}$. The lysate was treated with biotinylated-dRP or biotin as control for $1 \mathrm{~h}$ under rotation at $4^{\circ} \mathrm{C}$. Streptavidin beads were added to samples, which were further rotated for $2 \mathrm{~h}$ at $4^{\circ} \mathrm{C}$. Samples were boiled, loaded on to SDS-PAGE gels, and resulting blots probed for $\operatorname{NOX} 1,2$, and 4 and for $\beta$-actin as loading control.

\section{Intracellular free thiol monitoring by cysteine labeling}

BIAM was used to label free thiols in cell lysates as previously described (12). Cell lysates were obtained by sonication in anoxic conditions (cell lysis buffer: $150 \mathrm{~m} M \mathrm{NaCl}$, $0.5 \% \mathrm{v} / \mathrm{v}$ Triton-X, $50 \mathrm{~m} M$ Tris- $\mathrm{HCl}, \mathrm{pH} 6.5$, Complete $^{\mathrm{TM}}$ protease inhibitors). Cell lysates were labeled for $2 \mathrm{~h}$ with $20 \mu M$ BIAM in anoxic conditions. BIAM was then quenched with $10 \mathrm{~m} M \beta$-mercaptoethanol. Cell proteins were separated by SDS-PAGE, stained with streptavidin-HRP, and visualized by enhanced chemiluminescence.

Alternatively, VitaBright $-43^{\mathrm{TM}}$ was used to label free thiol groups on proteins (59). This reagent is cell permeable and gives thioester-coupled fluorescent products in a quantitative manner, which allows an estimate of cell oxidative state; $5 \times 10^{6}$ cells $/ \mathrm{ml}$ treated as described were labeled with VitaBright-43 and propidium iodide, as suggested by suppliers. NucleoCounter3000 (Chemometec A/S) was utilized to analyze cell fluorescence levels associated with the two dyes.

\section{Flow cytometry analysis of apoptosis}

Staining with Annexin V-fluorescein isothiocyanate (FITC) to detect cell surface exposure of phosphatidylserine was performed to examine apoptosis in cultured HUVECs treated with or without dRP for $24 \mathrm{~h}$ (50). During apoptosis, an early and ubiquitous event is the exposure of phosphatidylserine at the cell surface, which is detected with Annexin V-FITC. Cells treated with $5 \mathrm{~m} M$ diethyl maleate for $24 \mathrm{~h}$ served as a proapoptopic control. After incubation, cells were harvested with the gentle dissociating buffer TrypLE ${ }^{\circledR}$, pelleted by centrifugation, washed with PBS, and resuspended in
PBS. Cell suspensions were stained with Annexin V/FITC according to the manufacturer's instructions. Briefly, PBSwashed cells were suspended in $100 \mu \mathrm{l}$ FITC binding buffer at a minimum concentration of $1 \times 10^{6}$ combined with $5 \mu \mathrm{l}$ Annexin V/FITC. After $15 \mathrm{~min}$ of incubation in the dark on ice, cells were centrifuged at $2000 \mathrm{rpm}$ for $10 \mathrm{~min}$, resuspended in PBS, and analyzed using FACSCanto II (BD Biosciences). Cells that were Annexin $\mathrm{V}$ negative were considered viable cells. Cells positive for Annexin V were considered apoptotic. All samples were prepared in triplicate.

\section{Mouse wound healing}

Mouse maintenance and experimental procedures were performed according to local ethics approval and a dedicated UK Home Office Project license. Wounding was performed as described by Mahdipour and Mace (36). dRP pellets were produced by diluting dRP into PBS:methyl cellulose (1\%; $1: 1)$. Fifty microliters of this solution was then spotted and dried to form a pellet containing $10.3 \mu \mathrm{g}(25 \mathrm{nmol})$ of $\mathrm{dRP}$. Control pellets were generated in the same way by adding vehicle solution (PBS) instead of dRP. Pellets were administered immediately following wounding and rehydrated directly into the wound. Pellets were subsequently administered every other day on days 2,4 , and 6 . Wounds were harvested at day 7, as appropriate, from sacrificed animals with a 2-mm border, fixed in formalin, and embedded in paraffin. Tissues were sectioned using a Leica CM3050 S to produce serial sections of $10-\mu \mathrm{m}$ thickness. Following dewaxing in xylene/EtOH/MetOH, sections were stained with anti-VEGFR2/KDR/Flk-1 antibodies (1:100, No. AF644; R\&D Systems) and FITC-labeled anti-goat IgG secondary antibodies (1:200; Life Technologies). Images were captured using an EVOS FL microscope with a 4x/0.1 Plan-Achromat objective (red fluorescence channel and phase contrast). Alternatively, tissue sections were cut at $5-\mu \mathrm{m}$ thickness and stained with hematoxylin/eosin using Mayer's method (20). Sections were evaluated for vascularity by imaging using an Olympus CKX41 microscope with UPlanFl 4x/0.13 objective.

\section{Matrigel plug vascularization assay}

Mouse maintenance and experimental procedures were performed according to local ethics approval and a dedicated UK Home Office Project license. Wild-type (C57BL6/J) and $\mathrm{NOX}^{-1-}$ (B6.129S-Cybbtm1Din/J; Jackson Laboratories) mice were injected with $200 \mu \mathrm{l}$ of growth factor-reduced Matrigel (Corning) containing $41.2 \mu \mathrm{g}(100 \mathrm{nmol})$ of dRP or an equivalent volume of vehicle solution (PBS). Seven days after injection, the animals were euthanized and the Matrigel plug was explanted, fixed in $10 \%$ formalin, and embedded in paraffin. Tissues were sectioned using a Leica CM3050 S to produce serial sections of $50-\mu \mathrm{m}$ thickness. Following dewaxing in xylene/EtOH/MetOH, sections were stained either with hematoxylin or anti-CD31 antibodies (1:100, No. 3528; Cell Signaling Technologies), TRITC-labeled anti-mouse IgG secondary antibodies (1:200; Life Technologies), and $0.25 \mu \mathrm{g} / \mathrm{ml}$ DAPI. Images were captured using an EVOS FL microscope with a 4x/0.1 Plan-Achromat objective (red and blue fluorescence channels and phase contrast). Alternatively, tissue sections were cut at $5-\mu \mathrm{m}$ thickness and stained 
with hematoxylin using Mayer's method (20). Sections were evaluated for vascularity by imaging using an Olympus CKX41 microscope with UPlanFl 4x/0.13 objective.

\section{Acknowledgments}

This work was sponsored by the Biotechnology and Biological Sciences Research Council (BB/J002690/1), the Medical Research Council (MRC/CIC/2015), and the British Heart Foundation (PG/15/40/31522). The authors acknowledge Dr. Adrian Rogers for help with imaging and flow cytometry.

\section{Authors' Contributions}

D.V. and G.P. designed, performed, and analyzed experiments. J.M.W. performed chemistry experiments to conjugate dRP to agarose beads. T.M.F. performed qPCR experiments. H.M. provided expertise and logistics for HUVEC isolation. M.B. and K.M. performed experiments for the quantification of $\mathrm{dRP}$ in macrophages. S.R. and A.L. performed LC-MS for dRP quantification. C.P.D.W.-J. was involved in the initial conception of the study, provided expertise for HUVEC isolation and assessment of angiogenesis in vitro, and commented on the manuscript. G.P. wrote the manuscript.

\section{Author Disclosure Statement}

No competing financial interests exist.

\section{References}

1. Ahluwalia A and Tarnawski AS. Critical role of hypoxia sensor-HIF-1alpha in VEGF gene activation. Implications for angiogenesis and tissue injury healing. Curr Med Chem 19: 90-97, 2012.

2. Alig SK, Stampnik Y, Pircher J, Rotter R, Gaitzsch E, Ribeiro A, Wornle M, Krotz F, and Mannell H. The tyrosine phosphatase SHP-1 regulates hypoxia inducible factor1alpha (HIF-1alpha) protein levels in endothelial cells under hypoxia. PLoS One 10: e0121113, 2015.

3. Arbiser JL, Petros J, Klafter R, Govindajaran B, McLaughlin ER, Brown LF, Cohen C, Moses M, Kilroy S, Arnold RS, and Lambeth JD. Reactive oxygen generated by Nox 1 triggers the angiogenic switch. Proc Natl Acad Sci U S A 99: 715-720, 2002.

4. BelAiba RS, Djordjevic T, Petry A, Diemer K, Bonello S, Banfi B, Hess J, Pogrebniak A, Bickel C, and Gorlach A. NOX5 variants are functionally active in endothelial cells. Free Radic Biol Med 42: 446-459, 2007.

5. Bijnsdorp IV, Azijli K, Jansen EE, Wamelink MM, Jakobs C, Struys EA, Fukushima M, Kruyt FA, and Peters GJ. Accumulation of thymidine-derived sugars in thymidine phosphorylase overexpressing cells. Biochem Pharmacol 80: 786-792, 2010.

6. Brown NS and Bicknell R. Thymidine phosphorylase, 2-deoxy-D-ribose and angiogenesis. Biochem J $334(\mathrm{Pt} 1)$ : $1-8,1998$.

7. Brown NS, Jones A, Fujiyama C, Harris AL, and Bicknell R. Thymidine phosphorylase induces carcinoma cell oxidative stress and promotes secretion of angiogenic factors. Cancer Res 60: 6298-6302, 2000.

8. Carnesecchi S, Carpentier JL, Foti M, and Szanto I. Insulininduced vascular endothelial growth factor expression is mediated by the NADPH oxidase NOX3. Exp Cell Res 312: 3413-3424, 2006.

9. Cayatte AJ, Rupin A, Oliver-Krasinski J, Maitland K, Sansilvestri-Morel P, Boussard MF, Wierzbicki M, Verbeuren TJ, and Cohen RA. S17834, a new inhibitor of cell adhesion and atherosclerosis that targets NADPH oxidase. Arterioscler Thromb Vasc Biol 21: 1577-1584, 2001.

10. Chan EC, van Wijngaarden P, Chan E, Ngo D, Wang JH, Peshavariya HM, Dusting GJ, and Liu GS. NADPH oxidase 2 plays a role in experimental corneal neovascularization. Clin Sci (Lond) 130: 683-696, 2016.

11. Chandel NS, Trzyna WC, McClintock DS, and Schumacker PT. Role of oxidants in NF-kappa B activation and TNFalpha gene transcription induced by hypoxia and endotoxin. J Immunol 165: 1013-1021, 2000.

12. Choi KS, Park SY, Baek SH, Dey-Rao R, Park YM, Zhang H, Ip C, Park EM, Kim YH, and Park JH. Analysis of protein redox modification by hypoxia. Prep Biochem Biotechnol 36: 65-79, 2006.

13. Craige SM, Chen K, Pei Y, Li C, Huang X, Chen C, Shibata R, Sato K, Walsh K, Keaney JF, Jr. NADPH oxidase 4 promotes endothelial angiogenesis through endothelial nitric oxide synthase activation. Circulation 124: 731-740, 2011.

14. Csanyi G, Cifuentes-Pagano E, Al Ghouleh I, Ranayhossaini DJ, Egana L, Lopes LR, Jackson HM, Kelley EE, and Pagano PJ. Nox2 B-loop peptide, Nox2ds, specifically inhibits the NADPH oxidase Nox2. Free Radic Biol Med 51: 1116-1125, 2011.

15. Datla SR, Peshavariya H, Dusting GJ, Mahadev K, Goldstein BJ, and Jiang F. Important role of Nox4 type NADPH oxidase in angiogenic responses in human microvascular endothelial cells in vitro. Arterioscler Thromb Vasc Biol 27: 2319-2324, 2007.

16. Dickinson BC and Chang CJ. Chemistry and biology of reactive oxygen species in signaling or stress responses. Nat Chem Biol 7: 504-511, 2011.

17. Dong F, Zhou X, Li C, Yan S, Deng X, Cao Z, Li L, Tang B, Allen TD, and Liu J. Dihydroartemisinin targets VEGFR2 via the NF-kappaB pathway in endothelial cells to inhibit angiogenesis. Cancer Biol Ther 15: 1479-1488, 2014.

18. Drummond GR, Selemidis S, Griendling KK, and Sobey CG. Combating oxidative stress in vascular disease: NADPH oxidases as therapeutic targets. Nat Rev Drug Discov 10: 453-471, 2011.

19. Evangelista AM, Thompson MD, Bolotina VM, Tong X, and Cohen RA. Nox4- and Nox2-dependent oxidant production is required for VEGF-induced SERCA cysteine674 S-glutathiolation and endothelial cell migration. Free Radic Biol Med 53: 2327-2334, 2012.

20. Fischer AH, Jacobson KA, Rose J, and Zeller R. Hematoxylin and eosin staining of tissue and cell sections. $\mathrm{CSH}$ Protoc 2008: pdb.prot4986, 2008.

21. Gauuan PJ, Trova MP, Gregor-Boros L, Bocckino SB, Crapo JD, and Day BJ. Superoxide dismutase mimetics: Synthesis and structure-activity relationship study of MnTBAP analogues. Bioorg Med Chem 10: 3013-3021, 2002.

22. Guzy RD, Hoyos B, Robin E, Chen H, Liu L, Mansfield KD, Simon MC, Hammerling U, and Schumacker PT. Mitochondrial complex III is required for hypoxia-induced ROS production and cellular oxygen sensing. Cell Metab 1: 401-408, 2005.

23. Hensley K, Robinson KA, Gabbita SP, Salsman S, and Floyd RA. Reactive oxygen species, cell signaling, 
and cell injury. Free Radic Biol Med 28: 1456-1462, 2000.

24. Hotchkiss KA, Ashton AW, Klein RS, Lenzi ML, Zhu GH, and Schwartz EL. Mechanisms by which tumor cells and monocytes expressing the angiogenic factor thymidine phosphorylase mediate human endothelial cell migration. Cancer Res 63: 527-533, 2003.

25. Houliston RA, Pearson JD, and Wheeler-Jones CP. Agonist-specific cross talk between ERKs and p38(mapk) regulates PGI(2) synthesis in endothelium. Am J Physiol Cell Physiol 281: C1266-C1276, 2001.

26. Ikeda S, Yamaoka-Tojo M, Hilenski L, Patrushev NA, Anwar GM, Quinn MT, and Ushio-Fukai M. IQGAP1 regulates reactive oxygen species-dependent endothelial cell migration through interacting with Nox2. Arterioscler Thromb Vasc Biol 25: 2295-2300, 2005.

27. Ishikawa F, Miyazono K, Hellman U, Drexler H, Wernstedt C, Hagiwara K, Usuki K, Takaku F, Risau W, and Heldin $\mathrm{CH}$. Identification of angiogenic activity and the cloning and expression of platelet-derived endothelial cell growth factor. Nature 338: 557-562, 1989.

28. Jiang BH, Semenza GL, Bauer C, and Marti HH. Hypoxiainducible factor 1 levels vary exponentially over a physiologically relevant range of $\mathrm{O}_{2}$ tension. Am J Physiol 271: C1172-C1180, 1996.

29. Kaiser N, Sasson S, Feener EP, Boukobza-Vardi N, Higashi S, Moller DE, Davidheiser S, Przybylski RJ, and King GL. Differential regulation of glucose transport and transporters by glucose in vascular endothelial and smooth muscle cells. Diabetes 42: 80-89, 1993.

30. Kang KW, Lee SJ, and Kim SG. Molecular mechanism of nrf2 activation by oxidative stress. Antioxid Redox Signal 7: 1664-1673, 2005.

31. Kawahara A, Hattori S, Akiba J, Nakashima K, Taira T, Watari K, Hosoi F, Uba M, Basaki Y, Koufuji K, Shirouzu K, Akiyama S, Kuwano M, Kage M, and Ono M. Infiltration of thymidine phosphorylase-positive macrophages is closely associated with tumor angiogenesis and survival in intestinal type gastric cancer. Oncol Rep 24: 405-415, 2010.

32. Khatri JJ, Johnson C, Magid R, Lessner SM, Laude KM, Dikalov SI, Harrison DG, Sung HJ, Rong Y, and Galis ZS. Vascular oxidant stress enhances progression and angiogenesis of experimental atheroma. Circulation 109: 520 525, 2004.

33. Krishna MC, Samuni A, Taira J, Goldstein S, Mitchell JB, and Russo A. Stimulation by nitroxides of catalase-like activity of hemeproteins. Kinetics and mechanism. $J$ Biol Chem 271: 26018-26025, 1996.

34. Latham Birt SH, Purcell R, Botham KM, and WheelerJones CP. Endothelial HO-1 induction by model TG-rich lipoproteins is regulated through a NOX4-Nrf2 pathway. $J$ Lipid Res 57: 1204-1218, 2016.

35. Liekens S, Hernandez AI, Ribatti D, De Clercq E, Camarasa MJ, Perez-Perez MJ, and Balzarini J. The nucleoside derivative 5'-O-trityl-inosine (KIN59) suppresses thymidine phosphorylase-triggered angiogenesis via a noncompetitive mechanism of action. J Biol Chem 279: 29598-29605, 2004.

36. Mahdipour E and Mace KA. Analyzing the angiogenic potential of $\mathrm{Gr}-1(+) \mathrm{CD} 11 \mathrm{~b}(+)$ immature myeloid cells from murine wounds. Methods Mol Biol 916: 219-229, 2012.
37. Matono R, Miyano $\mathrm{K}$, Kiyohara $\mathrm{T}$, and Sumimoto $\mathrm{H}$. Arachidonic acid induces direct interaction of the p67(phox)-Rac complex with the phagocyte oxidase Nox2, leading to superoxide production. J Biol Chem 289: 24874 24884, 2014.

38. Matsushita S, Nitanda T, Furukawa T, Sumizawa T, Tani A, Nishimoto K, Akiba S, Miyadera K, Fukushima M, Yamada Y, Yoshida H, Kanzaki T, and Akiyama S. The effect of a thymidine phosphorylase inhibitor on angiogenesis and apoptosis in tumors. Cancer Res 59: 19111916, 1999.

39. Menden H, Tate E, Hogg N, and Sampath V. LPSmediated endothelial activation in pulmonary endothelial cells: Role of Nox2-dependent IKK-beta phosphorylation. Am J Physiol Lung Cell Mol Physiol 304: L445L455, 2013.

40. Menden H, Welak S, Cossette S, Ramchandran R, and Sampath V. Lipopolysaccharide (LPS)-mediated angiopoietin-2-dependent autocrine angiogenesis is regulated by NADPH oxidase 2 (Nox2) in human pulmonary microvascular endothelial cells. J Biol Chem 290: 54495461, 2015.

41. Meng D, Mei A, Liu J, Kang X, Shi X, Qian R, and Chen S. NADPH oxidase 4 mediates insulin-stimulated HIF-1alpha and VEGF expression, and angiogenesis in vitro. PLoS One 7: e48393, 2012.

42. Miyadera K, Sumizawa T, Haraguchi M, Yoshida H, Konstanty W, Yamada Y, and Akiyama S. Role of thymidine phosphorylase activity in the angiogenic effect of platelet derived endothelial cell growth factor/thymidine phosphorylase. Cancer Res 55: 1687-1690, 1995.

43. Morgan MJ and Liu ZG. Crosstalk of reactive oxygen species and NF-kappaB signaling. Cell Res 21: 103-115, 2011.

44. Naula CM, Logan FJ, Wong PE, Barrett MP, and Burchmore RJ. A glucose transporter can mediate ribose uptake: Definition of residues that confer substrate specificity in a sugar transporter. J Biol Chem 285: 29721-29728, 2010.

45. Pendyala S, Gorshkova IA, Usatyuk PV, He D, Pennathur A, Lambeth JD, Thannickal VJ, and Natarajan V. Role of Nox4 and Nox 2 in hyperoxia-induced reactive oxygen species generation and migration of human lung endothelial cells. Antioxid Redox Signal 11: 747-764, 2009.

46. Peshavariya HM, Chan EC, Liu GS, Jiang F, and Dusting GJ. Transforming growth factor-beta1 requires NADPH oxidase 4 for angiogenesis in vitro and in vivo. $J$ Cell Mol Med 18: 1172-1183, 2014.

47. Pugh CW and Ratcliffe PJ. Regulation of angiogenesis by hypoxia: Role of the HIF system. Nat Med 9: 677-684, 2003.

48. Pugmire MJ and Ealick SE. Structural analyses reveal two distinct families of nucleoside phosphorylases. Biochem J 361: 1-25, 2002.

49. Pula G, Garonna E, Dunn WB, Hirano M, Pizzorno G, Campanella M, Schwartz EL, El Kouni MH, and WheelerJones CP. Paracrine stimulation of endothelial cell motility and angiogenesis by platelet-derived deoxyribose1-phosphate. Arterioscler Thromb Vasc Biol 30: 26312638, 2010.

50. Pula G, Mayr U, Evans C, Prokopi M, Vara DS, Yin X, Astroulakis Z, Xiao Q, Hill J, Xu Q, and Mayr M. Pro- 
teomics identifies thymidine phosphorylase as a key regulator of the angiogenic potential of colony-forming units and endothelial progenitor cell cultures. Circ Res 104: 3240, 2009.

51. Qutub AA and Popel AS. Reactive oxygen species regulate hypoxia-inducible factor 1alpha differentially in cancer and ischemia. Mol Cell Biol 28: 5106-5119, 2008.

52. Ray PD, Huang BW, and Tsuji Y. Reactive oxygen species (ROS) homeostasis and redox regulation in cellular signaling. Cell Signal 24: 981-990, 2012.

53. Renard P, Ernest I, Houbion A, Art M, Le Calvez H, Raes $\mathrm{M}$, and Remacle J. Development of a sensitive multi-well colorimetric assay for active NFkappaB. Nucleic Acids Res 29: E21, 2001.

54. Rey FE, Cifuentes ME, Kiarash A, Quinn MT, and Pagano PJ. Novel competitive inhibitor of NAD(P)H oxidase assembly attenuates vascular $\mathrm{O}(2)(-)$ and systolic blood pressure in mice. Circ Res 89: 408-414, 2001.

55. Ronicke V, Risau W, and Breier G. Characterization of the endothelium-specific murine vascular endothelial growth factor receptor-2 (Flk-1) promoter. Circ Res 79: 277-285, 1996.

56. Schreck R, Rieber P, and Baeuerle PA. Reactive oxygen intermediates as apparently widely used messengers in the activation of the NF-kappa B transcription factor and HIV1. EMBO J 10: 2247-2258, 1991.

57. Schroder K, Zhang M, Benkhoff S, Mieth A, Pliquett R, Kosowski J, Kruse C, Luedike P, Michaelis UR, Weissmann N, Dimmeler S, Shah AM, and Brandes RP. Nox 4 is a protective reactive oxygen species generating vascular NADPH oxidase. Circ Res 110: 1217-1225, 2012.

58. Sengupta S, Sellers LA, Matheson HB, and Fan TP. Thymidine phosphorylase induces angiogenesis in vivo and in vitro: An evaluation of possible mechanisms. $\mathrm{Br} \mathrm{J}$ Pharmacol 139: 219-231, 2003.

59. Skindersoe ME and Kjaerulff S. Comparison of three thiol probes for determination of apoptosis-related changes in cellular redox status. Cytometry A 85: 179-187, 2014.

60. Takakura Y, Kuentzel SL, Raub TJ, Davies A, Baldwin SA, and Borchardt RT. Hexose uptake in primary cultures of bovine brain microvessel endothelial cells. I. Basic characteristics and effects of D-glucose and insulin. Biochim Biophys Acta 1070: 1-10, 1991.

61. Tobe M, Isobe Y, Tomizawa H, Nagasaki T, Takahashi H, Fukazawa T, and Hayashi H. Discovery of quinazolines as a novel structural class of potent inhibitors of NF-kappa B activation. Bioorg Med Chem 11: 383-391, 2003.

62. Toi M, Atiqur Rahman M, Bando $\mathrm{H}$, and Chow LW. Thymidine phosphorylase (platelet-derived endothelial-cell growth factor) in cancer biology and treatment. Lancet Oncol 6: 158-166, 2005.

63. Tojo T, Ushio-Fukai M, Yamaoka-Tojo M, Ikeda S, Patrushev N, and Alexander RW. Role of gp91phox (Nox2)-containing $\mathrm{NAD}(\mathrm{P}) \mathrm{H}$ oxidase in angiogenesis in response to hindlimb ischemia. Circulation 111: 23472355, 2005.

64. Tozzi MG, Camici M, Mascia L, Sgarrella F, and Ipata PL. Pentose phosphates in nucleoside interconversion and catabolism. FEBS J 273: 1089-1101, 2006.

65. Ushio-Fukai M and Nakamura Y. Reactive oxygen species and angiogenesis: NADPH oxidase as target for cancer therapy. Cancer Lett 266: 37-52, 2008.
66. Vara D, Campanella M, and Pula G. The novel NOX inhibitor 2-acetylphenothiazine impairs collagen-dependent thrombus formation in a GPVI-dependent manner. $\mathrm{Br} \mathrm{J}$ Pharmacol 168: 212-224, 2013.

67. Vara DS, Campanella M, Canobbio I, Dunn WB, Pizzorno G, Hirano M, and Pula G. Autocrine amplification of integrin alphaIIbbeta3 activation and platelet adhesive responses by deoxyribose-1-phosphate. Thromb Haemost 109: 1108-1119, 2013.

68. Vigont V, Kolobkova Y, Skopin A, Zimina O, Zenin V, Glushankova L, and Kaznacheyeva E. Both Orai1 and TRPC1 are involved in excessive store-operated calcium entry in striatal neurons expressing mutant huntingtin exon 1. Front Physiol 6: 337, 2015.

69. Wang J, Hong Z, Zeng C, Yu Q, and Wang H. NADPH oxidase 4 promotes cardiac microvascular angiogenesis after hypoxia/reoxygenation in vitro. Free Radic Biol Med 69: 278-288, 2014.

70. Wei Y, Gong J, Xu Z, and Duh EJ. Nrf2 promotes reparative angiogenesis through regulation of NADPH oxidase-2 in oxygen-induced retinopathy. Free Radic Biol Med 99: 234-243, 2016.

71. Zafarullah M, Li WQ, Sylvester J, and Ahmad M. Molecular mechanisms of N-acetylcysteine actions. Cell Mol Life Sci 60: 6-20, 2003.

Address correspondence to: Dr. Giordano Pula

Institute of Biomedical and Clinical Science University of Exeter Medical School

Exeter EX1 2LU

United Kingdom

E-mail: g.pula@exeter.ac.uk

Date of first submission to ARS Central, August 19, 2016; date of final revised submission, July 8, 2017; date of acceptance, July 31, 2017.

$\begin{aligned} & \text { Abbreviations Used } \\ \mathrm{BIAM}=\text { biotinyl-iodoacetamide } & \\ \mathrm{CMH}= & \text { - } \text {-methoxycarbonyl-2,2,5,5- } \\ & \text { tetramethylpyrrolidine } \\ \mathrm{DAPI} & =4^{\prime}, 6 \text {-diamidino-2-phenylindole } \\ \mathrm{DHE} & =\text { dihydroethidium } \\ \mathrm{DMSO} & =\text { dimethyl sulfoxide } \\ \mathrm{DPI} & =\text { diphenylene iodonium } \\ \mathrm{dRP} & =\text { deoxyribose-1-phosphate } \\ \mathrm{ELISA} & =\text { enzyme-linked immunosorbent assay } \\ \mathrm{EPR} & =\text { electron paramagnetic resonance } \\ \mathrm{FBS} & =\text { fetal bovine serum } \\ \mathrm{FITC} & =\text { fluorescein isothiocyanate } \\ \mathrm{GFR} & =\text { growth factor-reduced } \\ \mathrm{GLUT} 1 & =\text { glucose transporter } 1 \\ \mathrm{HIF}-1 & =\text { hypoxia-induced factor } 1 \\ \mathrm{HRP} & =\text { horseradish peroxidase } \\ \mathrm{HUVES} & =\text { human umbilical vein endothelial cells } \\ \mathrm{IFN} \gamma & =\text { interferon gamma } \\ \mathrm{KHB} & =\text { Krebs HEPES buffer }\end{aligned}$




\section{Abbreviations Used (Cont.)}

LC-MS = liquid chromatography-mass spectrometry

LPS = lipopolysaccharide

MnTBAP $=\mathrm{Mn}(\mathrm{III})$ tetrakis(4-benzoic acid)porphyrin

mRNA $=$ messenger RNA

$\mathrm{NAC}=\mathrm{N}$-acetyl-L-cysteine

$\mathrm{NF}-\kappa \mathrm{B}=$ nuclear factor kappa $\mathrm{B}$

$\mathrm{NOX}=\mathrm{NADPH}$ oxidase

Nrf-2 = nuclear factor erythroid 2-related factor 2

$\mathrm{PBS}=$ phosphate-buffered saline

$\mathrm{PNP}=$ purine nucleoside phosphorylase

$\mathrm{QNZ}=$ N4-[2-(4-phenoxyphenyl)ethyl]-4,6quinazolinediamine
$\mathrm{qPCR}=$ real-time quantitative polymerase chain reaction

QTOF $=$ quadrupole time-of-flight

ROS $=$ reactive oxygen species

SDS-PAGE = sodium dodecyl sulfate-polyacrylamide gel electrophoresis

siRNA $=$ small interfering RNA

$\mathrm{SOD}=$ superoxide dismutase

Tempol = 4-hydroxy-2,2,6,6-tetramethylpiperidin1-oxyl

$\mathrm{TP}=$ thymidine phosphorylase

$\mathrm{UP}=$ uridine phosphorylase

$\mathrm{VEGF}=$ vascular endothelial growth factor

VEGFR2 $=$ VEGF receptor 2 